\title{
Multifunctional P450 Monooxygenase CftA Diversifies the Clifednamide Pool Through Tandem $\mathbf{C}-\mathrm{H}$ Bond Activations
}

\author{
Supporting Information
}

\begin{abstract}
Jinping Yang, ${ }^{\dagger}$ Y unci Qi, ${ }^{\dagger}$ Joshua A. V. Blodgett, ${ }^{\ddagger} *$ Timothy A. Wencewicz ${ }^{\dagger *}$
†Department of Chemistry, Washington University in St. Louis, One Brookings Drive, St. Louis, MO, 63130, USA.

Department of Biology, Washington University in St. Louis, One Brookings Drive, St. Louis, MO, 63130, USA.
\end{abstract}

Table of Contents

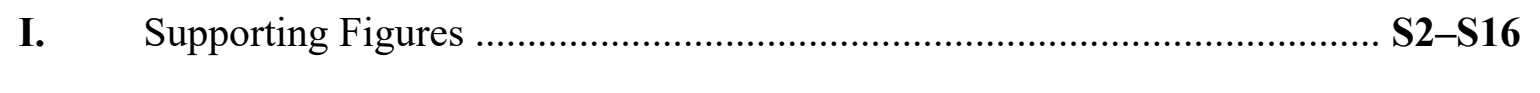

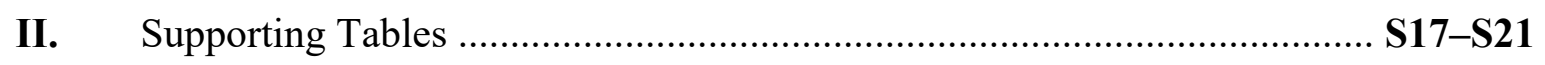

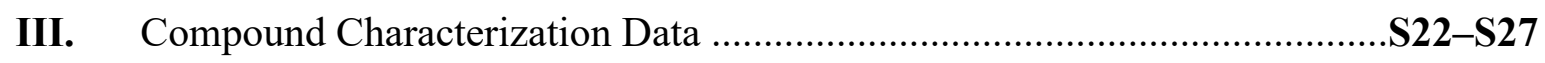

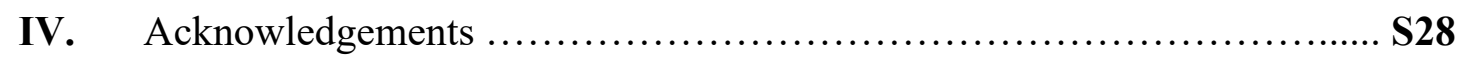

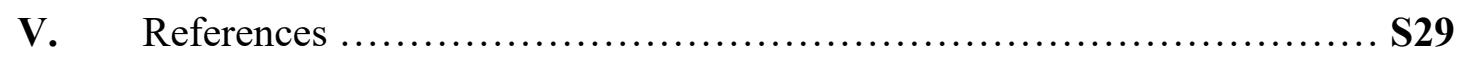

*To whom correspondence should be addressed:

Timothy A. Wencewicz

Email: wencewicz@wustl.edu

Phone: 314-935-7247

Fax: 314-935-4481

ORCID: 0000-0002-5839-6672
Joshua A.V. Blodgett

Email: jblodgett@wustl.edu

Phone: 314-935-6233

Fax: 314-935-4432

ORCID: 0000-0002-7080-5870 


\section{Supporting Figures}

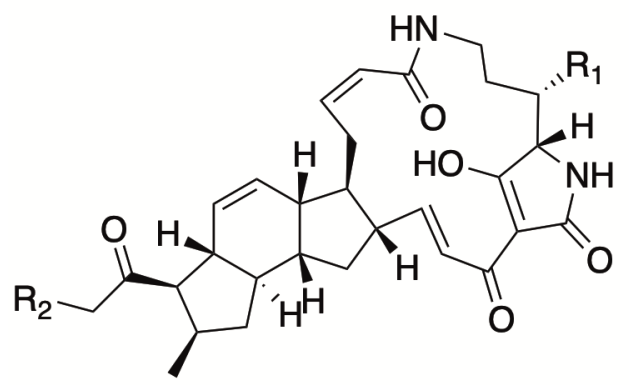

clifednamide $\mathrm{AR}_{1}=\mathrm{H}, \mathrm{R}_{2}=\mathrm{H}, 2$ clifednamide $B \mathrm{R}_{1}=\mathrm{OH}, \mathrm{R}_{2}=\mathrm{H}$ clifednamide $\mathrm{C} \mathrm{R}_{1}=\mathrm{H}, \mathrm{R}_{2}=\mathrm{OH}, 3$ clifednamdie $\mathrm{E} \mathrm{R}_{1}=\mathrm{OH}, \mathrm{R}_{2}=\mathrm{OH}$

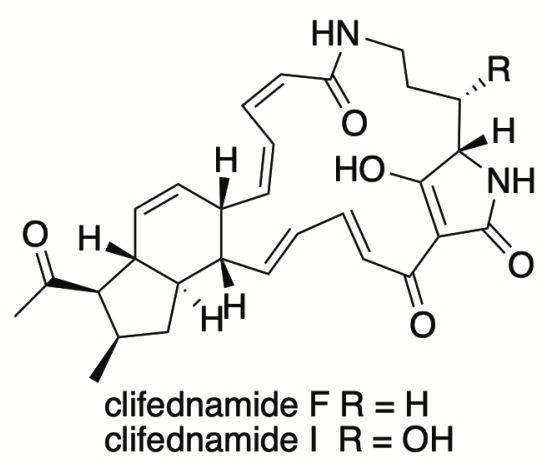<smiles>CC[C@H]1C(C)CC2[C@H]1C=C[C@H](/C=C/C=C\C(N)=O)[C@H]2/C=C/C=C/C(=O)C1=C(O)[C@@H](CCC(=O)O)NC1=O</smiles><smiles>CC(=O)[C@H]1C(C)CC2[C@H]1C=C[C@H]1[C@@H]2C[C@H](/C=C/C(=O)C2=C(O)[C@@H](CCC(N)=O)NC2=O)[C@H]1C/C=C\C(=O)O</smiles>

clifednamide D

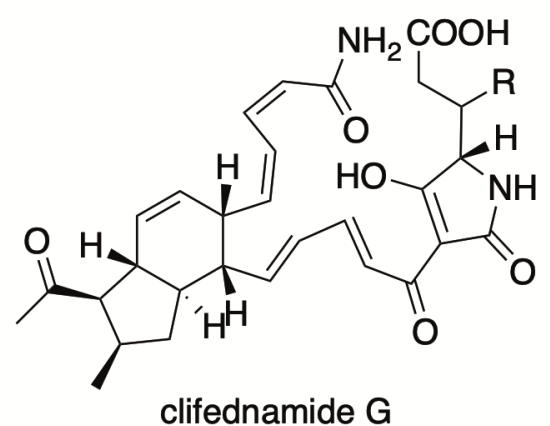

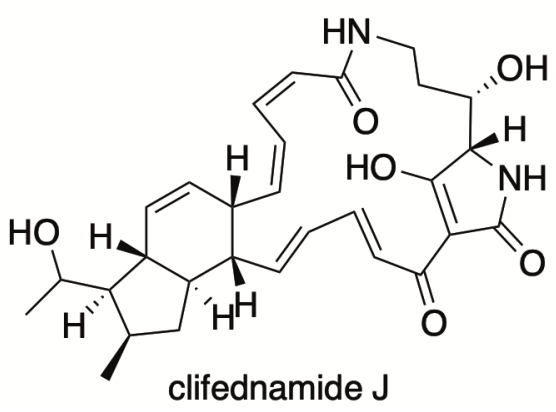

Figure S1. Structure of clifednamide A-J. Clifednamide A and B were characterized by Blodgett et al. ${ }^{1}$ Clifednamide D-J were recently reported by the Li group. The structure of clifednamide C, boxed in red, is elucidated in this work. 

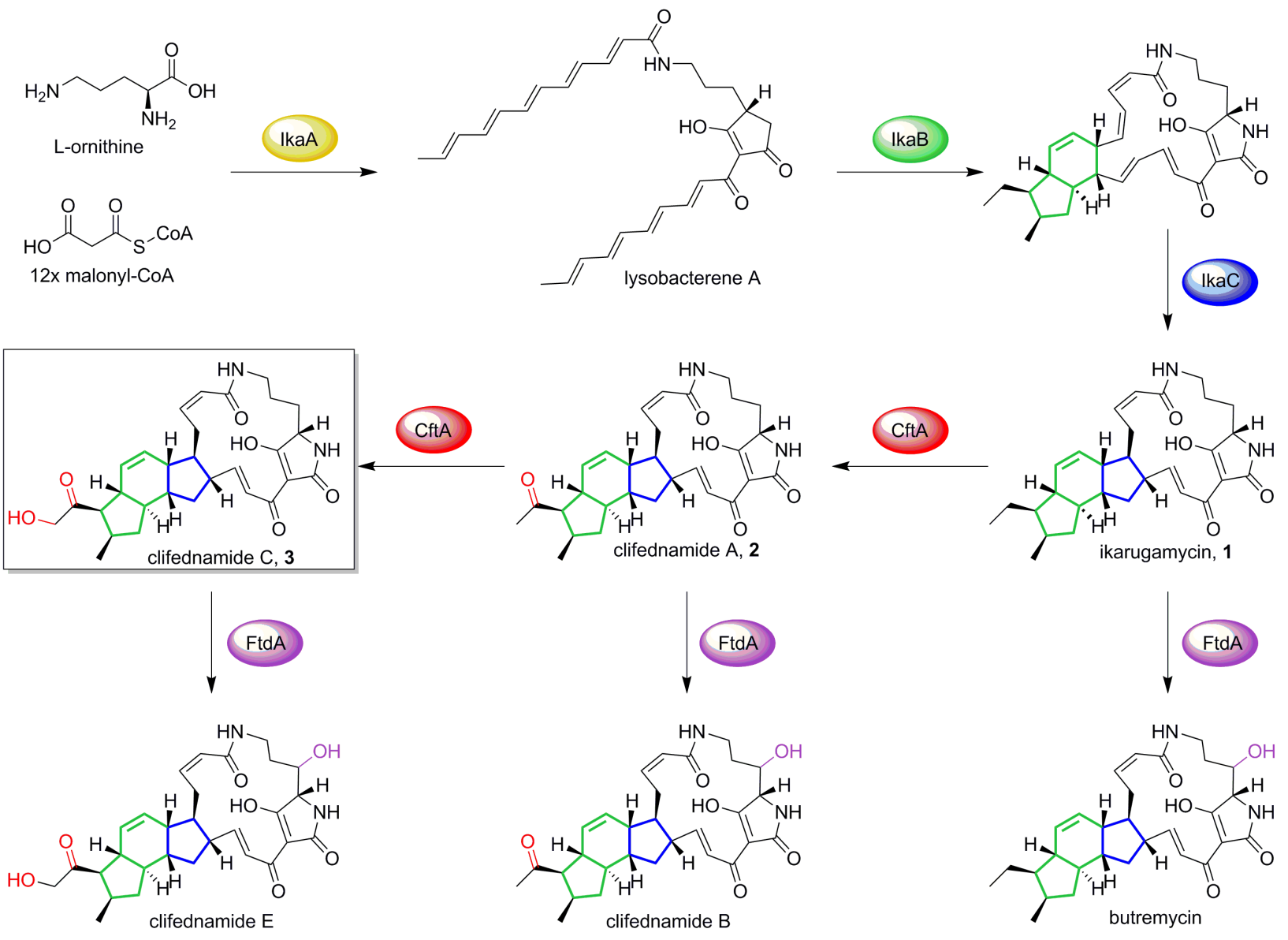

Figure S2. Biosynthesis of PTM natural products. L-Ornithine and malonyl-CoA are converted to lysobacterene A by the hybrid PKS-NRPS IkaA. The FAD oxidoreductase IkaB catalyzes the reductive cyclization to the fused 5-6 ring intermediate. $\mathrm{Zn}$-dependent alcohol dehydrogenase IkaC converts this intermediate to ikarugamycin which serves as a common intermediate on pathway to butremycin and clifednamides. The P450 CftA (characterized in this work) catalyzes sequential conversion of ikarugamycin to methyl ketone clifednamide A and hydroxymethyl ketone clifednamide C. Fatty acid desaturase FtdA hydroxylates the Orn-derived carbon chain of the macrolactam ring to complete the biosynthesis of clifednamide $\mathrm{E} / \mathrm{B}^{2}$ and butremycin ${ }^{3}$. 


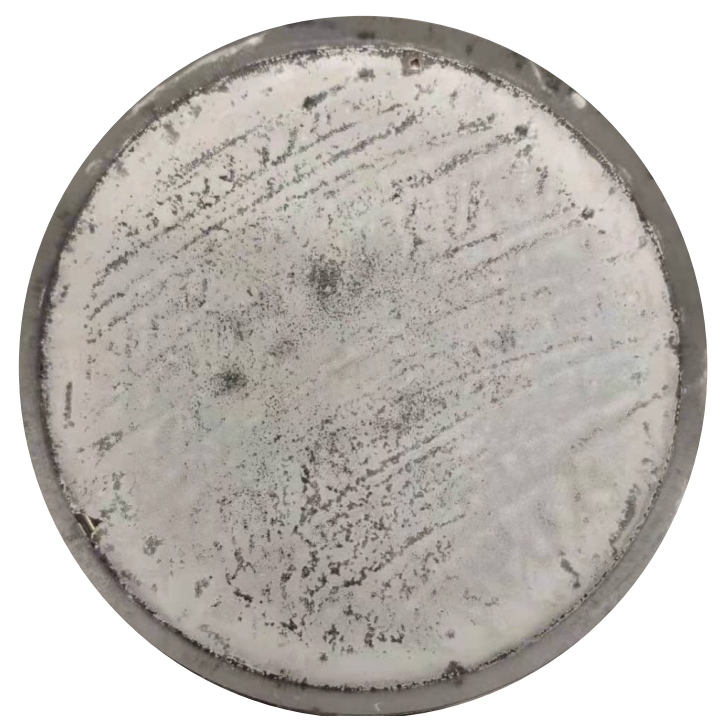

Figure S3. Image of Streptomyces sp. strain NRRL F-2890 JV772 ${ }^{1}$ grown on ATCC172 solid medium plate after 6 days at $28^{\circ} \mathrm{C}$. 


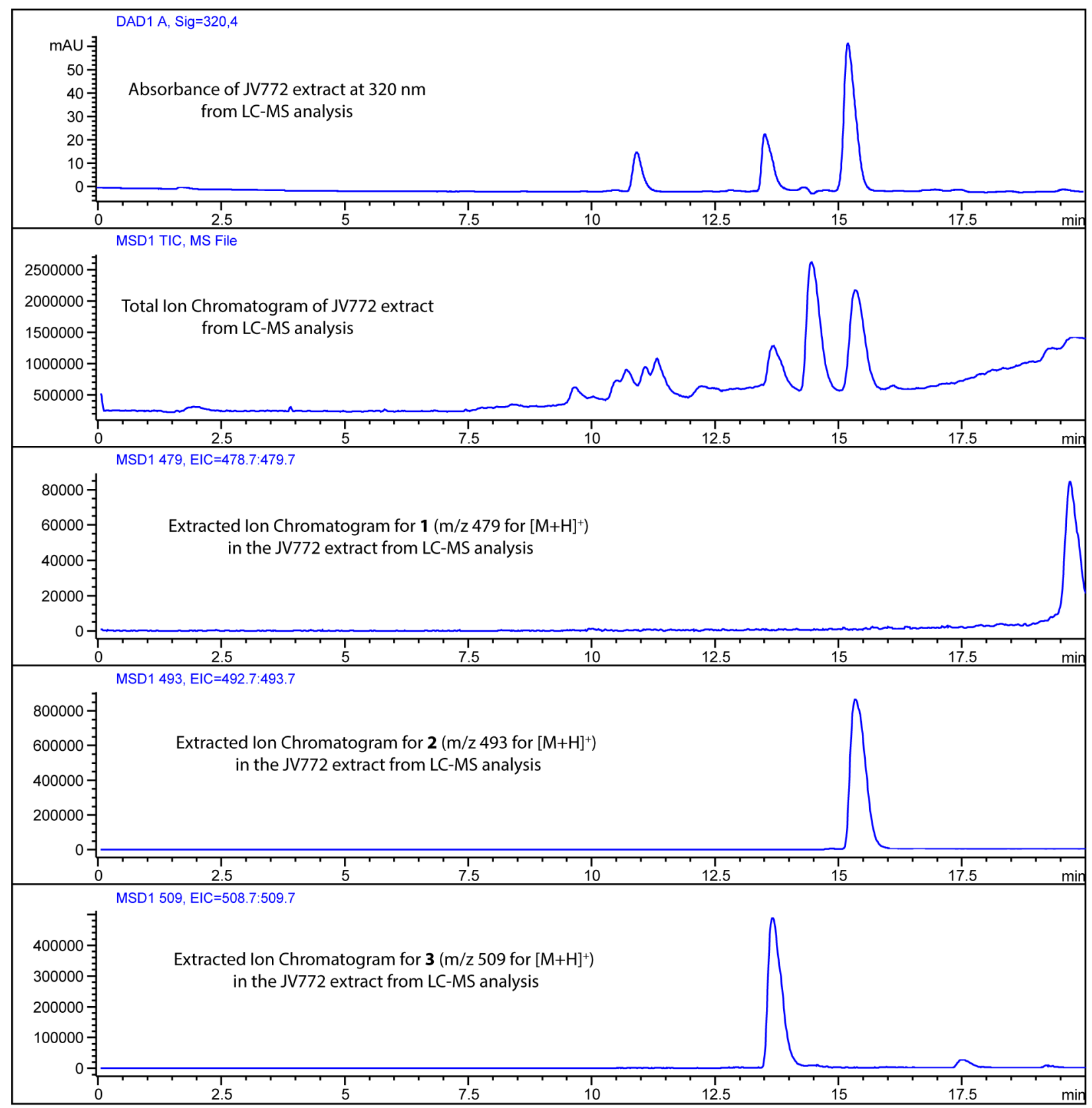

Figure S4. LC-MS analysis of culture extract from Streptomyces sp. strain NRRL F-2890 JV772 ${ }^{1}$ grown on solid ATCC172 medium after 6 s at $28{ }^{\circ} \mathrm{C}$. Agar plates like that shown in Figure S1 were soaked with ethyl acetate overnight and concentrated via rotary evaporation. The crude residue was dissolved in methanol and analyzed by low resolution LC-MS to generate the chromatograms provided. The y-axis on top chromatogram is the absorbance unit at $263 \mathrm{~nm}$. The $\mathrm{y}$-axes of other chromatograms represent ion counts for total and extracted ion chromatograms. The $\mathrm{x}$-axis for all chromatograms is retention time on the analytical RP-C18 column. 


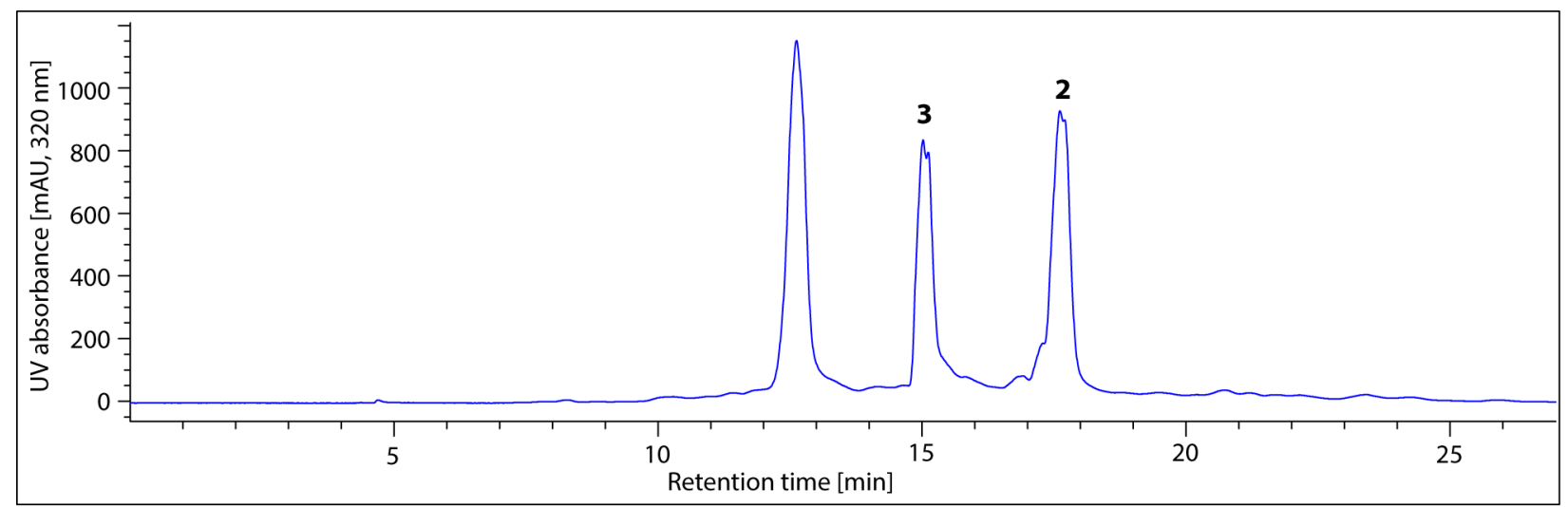

Figure S5. Prep-HPLC optical absorbance chromatogram at $320 \mathrm{~nm}$ for fractionation of Streptomyces sp. strain NRRL F- $2890 \mathrm{JV} 72^{1}$ culture extract concentrate using preparative scale RP-C18 column. Peaks corresponding to pure clifednamide C (3) and A (2) are indicated. 


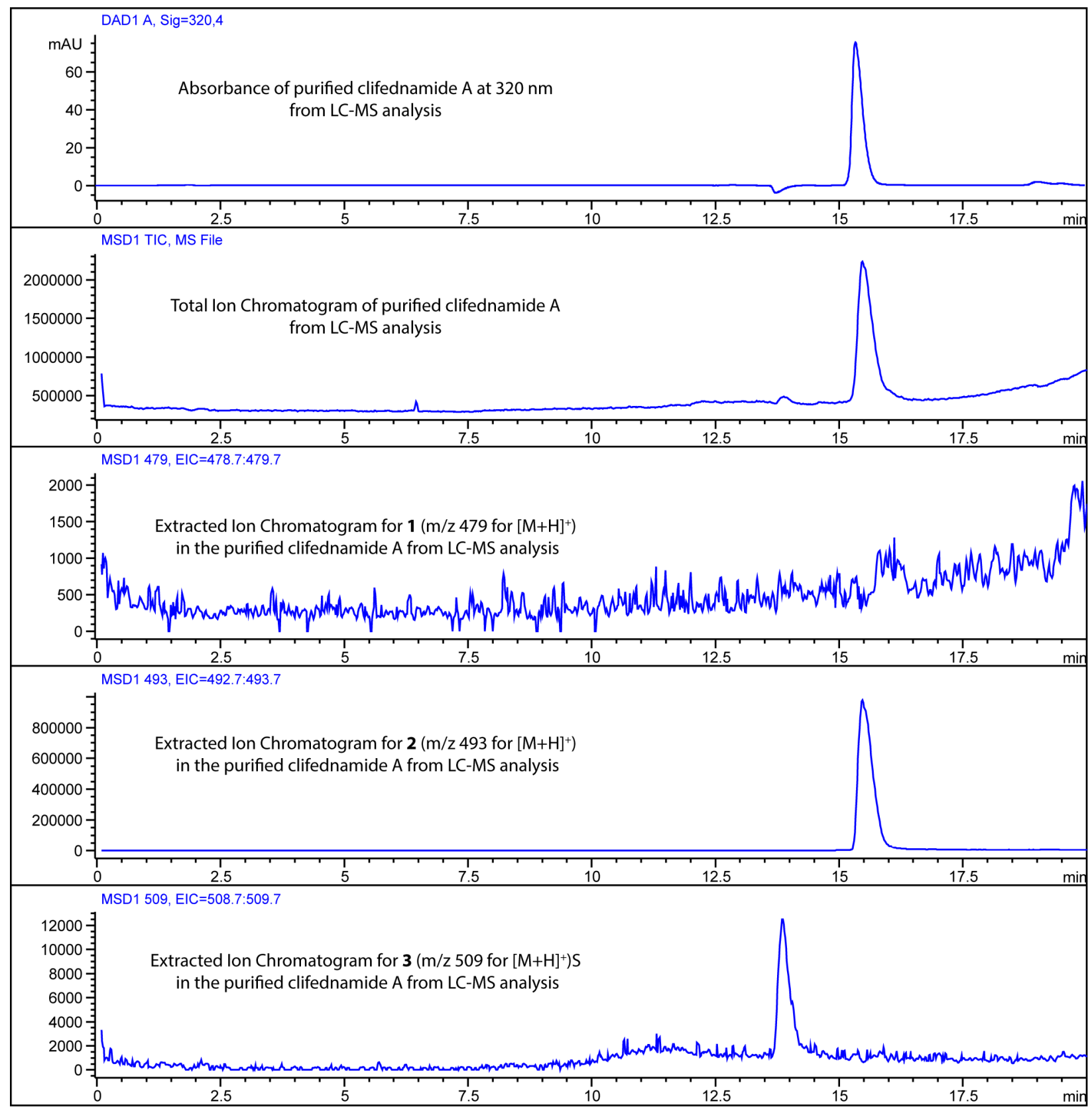

Figure S6. LC-MS analysis of purified clifednamide A after preparative HPLC purification from Streptomyces sp. strain NRRL F-2890 JV772 culture extract. 


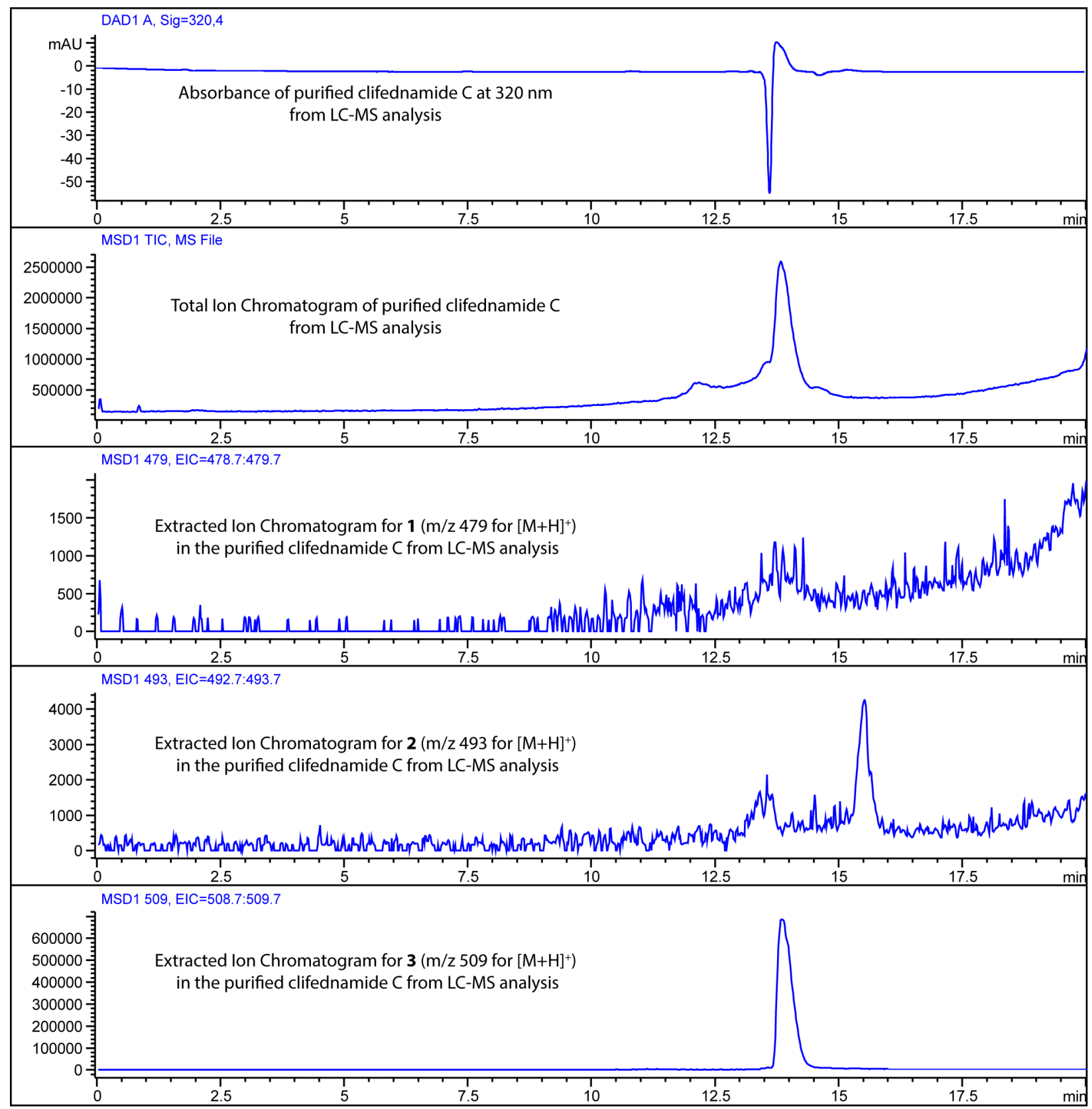

Figure S7. LC-MS analysis of purified clifednamide C after preparative HPLC purification from Streptomyces sp. strain NRRL F-2890 JV772 culture extract. 


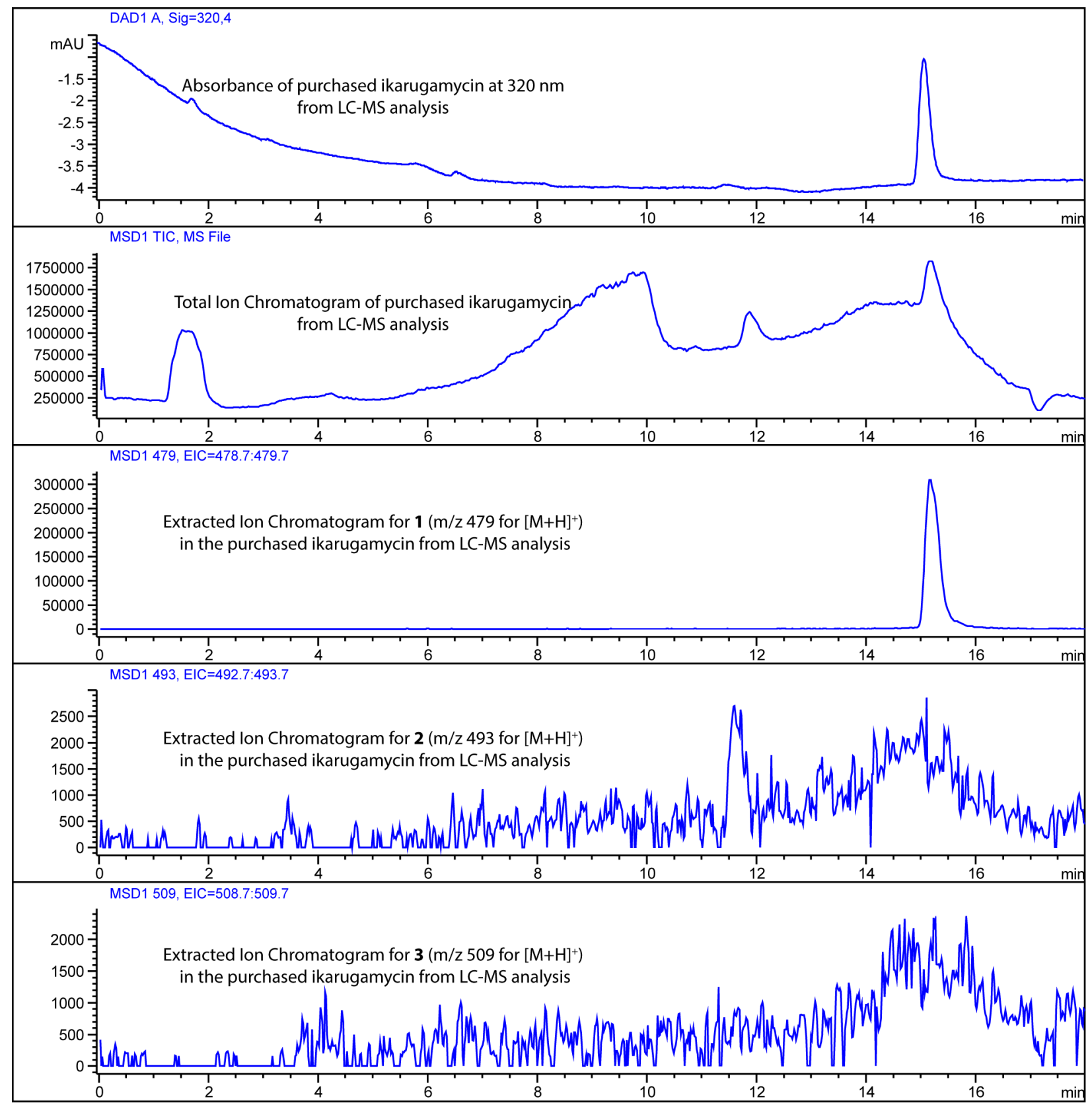

Figure S8. LC-MS analysis of ikarugamycin purchased from Santa Cruz Biotechnology. 
A.

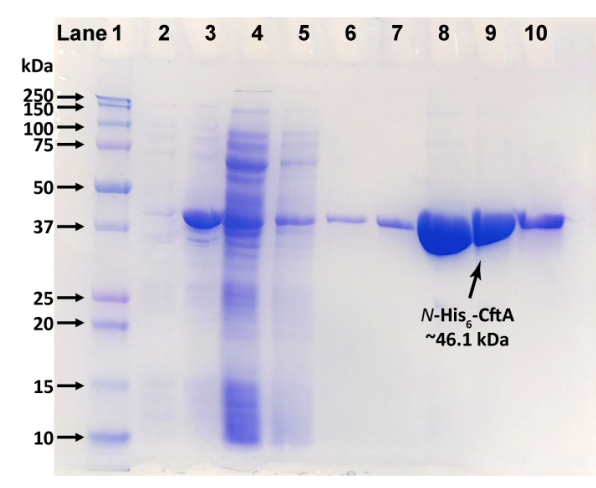

B.

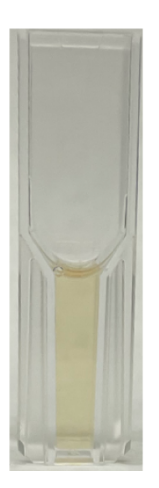

D.

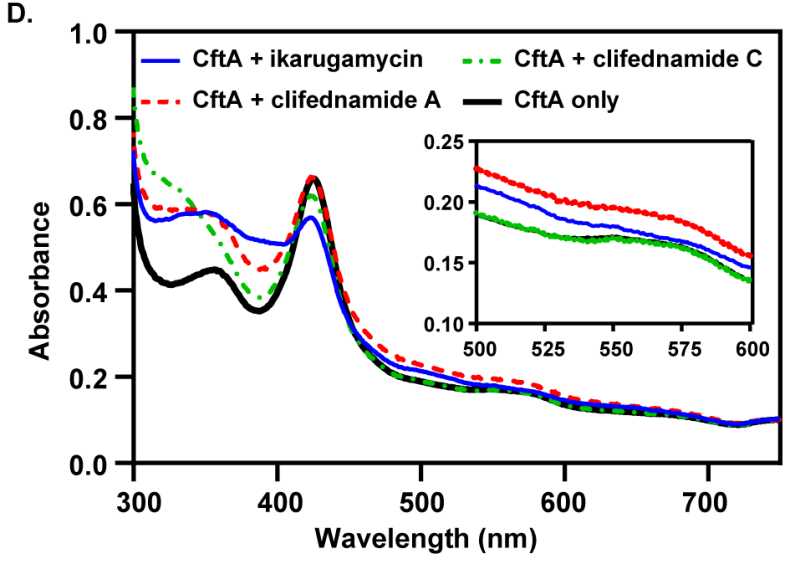

c.

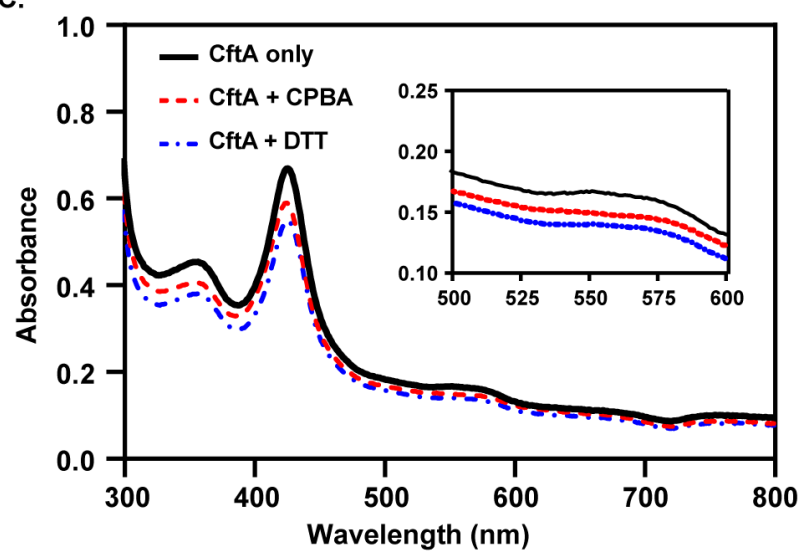

E.

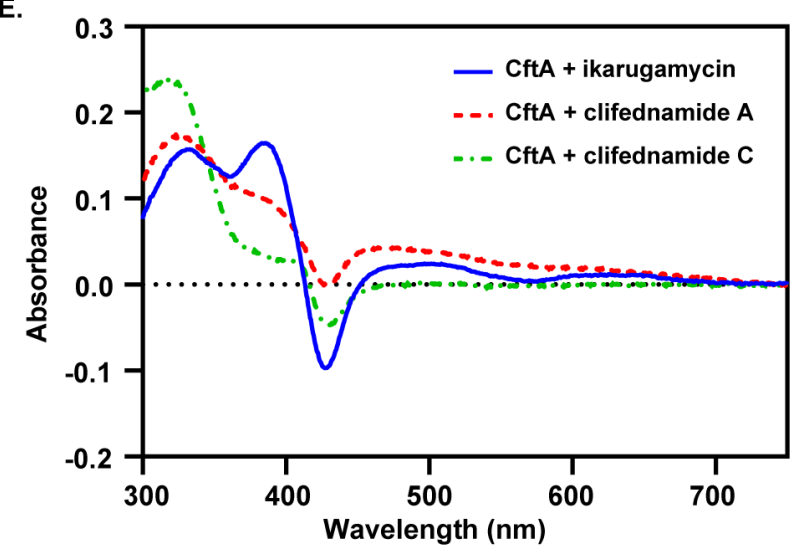

Figure S9. Biochemical characterization of recombinant $\mathrm{N}$-His6-tagged $\mathrm{CftA}$ under aerobic conditions. (A) SDS-PAGE analysis of pure CftA from E. coli Gold BL21. Lane 1, pre-stained protein ladder; Lane 2, uninduced cells; Lane 3, induced cells; Lane 4, flowthrough; Lane 5, wash 1; Lane 6, wash 2; Lane 7, elution 1; Lane 8, elution 2; Lane 9, elution 3; Lane 10, elution 4. During IMAC purification, Ni-NTA resin was overloaded with protein, thus a light band can be observed in the wash samples (lanes 6 and 7). (B) $60 \mu \mathrm{M}$ of CftA protein in $100 \mathrm{mM}$ potassium phosphate buffer at $\mathrm{pH} 7.0$ in a methacrylate cuvette. (C) UV-vis absorbance spectrum of $60 \mu \mathrm{M}$ of CftA in $100 \mathrm{mM}$ potassium phosphate buffer at $\mathrm{pH} 7.0$ in a quartz cuvette in the presence of oxidizing (mCPBA) or reducing (DTT) agent. The oxidized resting state of the CftA spectrum displays a peak with the major Soret band located at $425 \mathrm{~nm}$, and the alpha and beta absorption bands at 560 and $510 \mathrm{~nm}$, respectively. This supports that CftA in the absence of substrate was in the resting state or low spin form with a water molecule as the sixth proximal ligand to the iron of the heme group. ${ }^{4}$ (D) Optical absorbance spectra were recorded in $100 \mathrm{mM}$ potassium $\mathrm{P}_{\mathrm{i}}$ buffer (pH 7.0) w/ $30 \mu \mathrm{M} \mathrm{CftA}$, either with $135 \mu \mathrm{M}$ ikarugamycin (1, blue), $135 \mu \mathrm{M}$ clifednamide A (2, red), or $135 \mu \mathrm{M}$ clifednamide $\mathrm{C}$ (3, green) or without ligand (black). (E) Difference spectrum from mathematical subtraction of unliganded CftA (black spectrum, panel D) from that of each spectrum individually corresponding to ligand bound CftA (blue, red, and green spectra in panel D). 

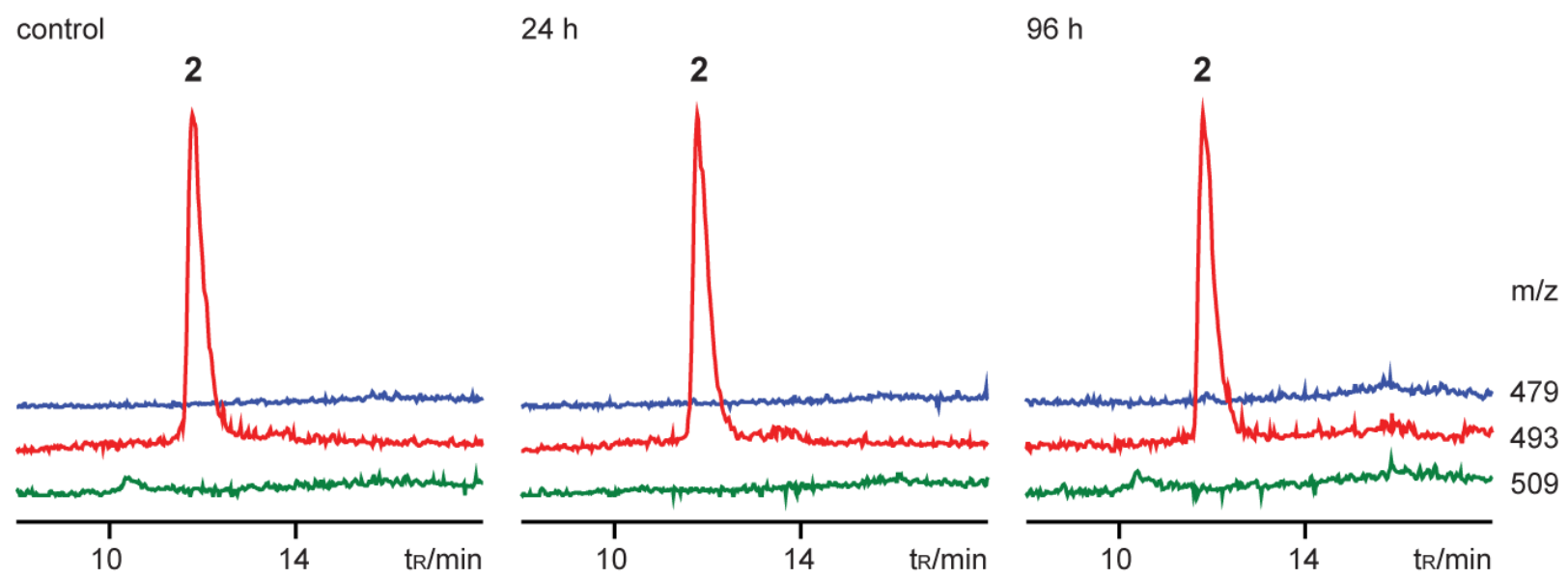

Figure S10. Extracted ion chromatograms (EICs) generated from low resolution single quadrupole LC-MS analyses of CftA reaction mixtures after $24 \mathrm{~h}$ and $96 \mathrm{~h}$ using clifednamide A as substrate. Control reaction contained no CftA. The $\mathrm{m} / \mathrm{z}$ values correspond to $[\mathrm{M}+\mathrm{H}]^{+}$molecular ions for ikarugamycin $(\mathbf{1}, 479)$, clifednamide A $(\mathbf{2}, 493)$, and clifednamide C $(\mathbf{3}, 509)$. The $\mathrm{x}$-axis represents retention time $\left(t_{R}\right)$. The y-axis represents relative EICs for target ions. No obvious clifednamide $\mathrm{C}$ (3) formation. 
A.
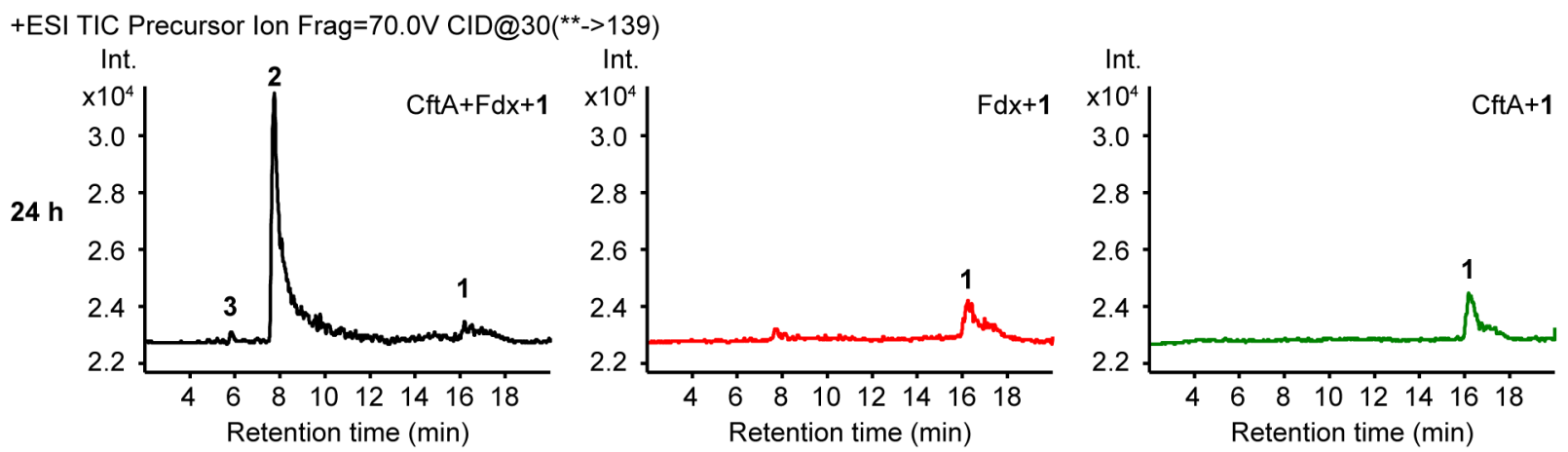

DAD1 - D: Sig:320, 8.0
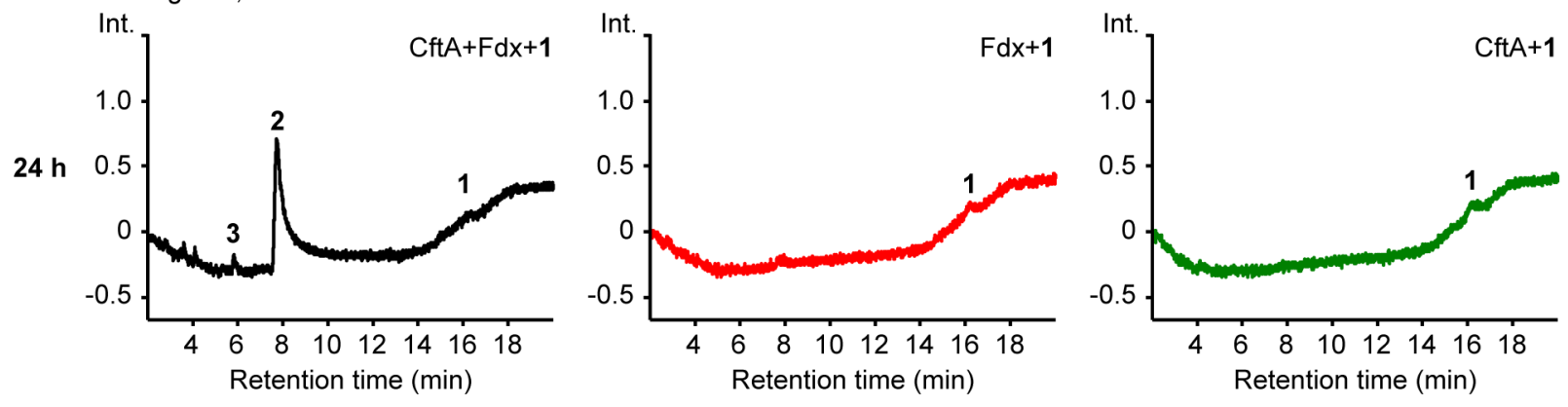

B.
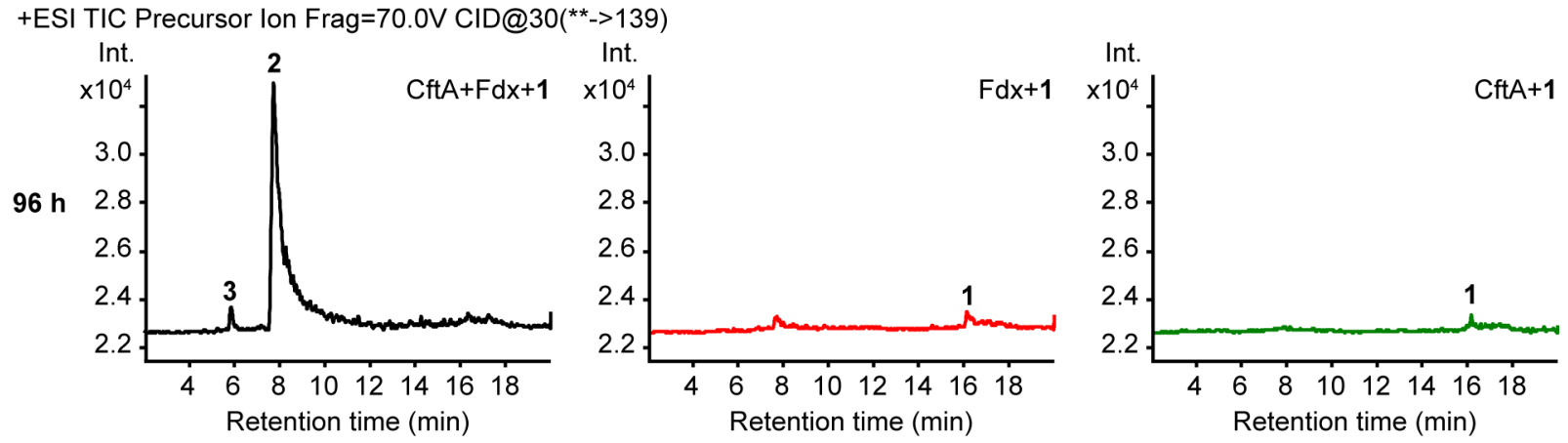

DAD1 - D: Sig:320, 8.0
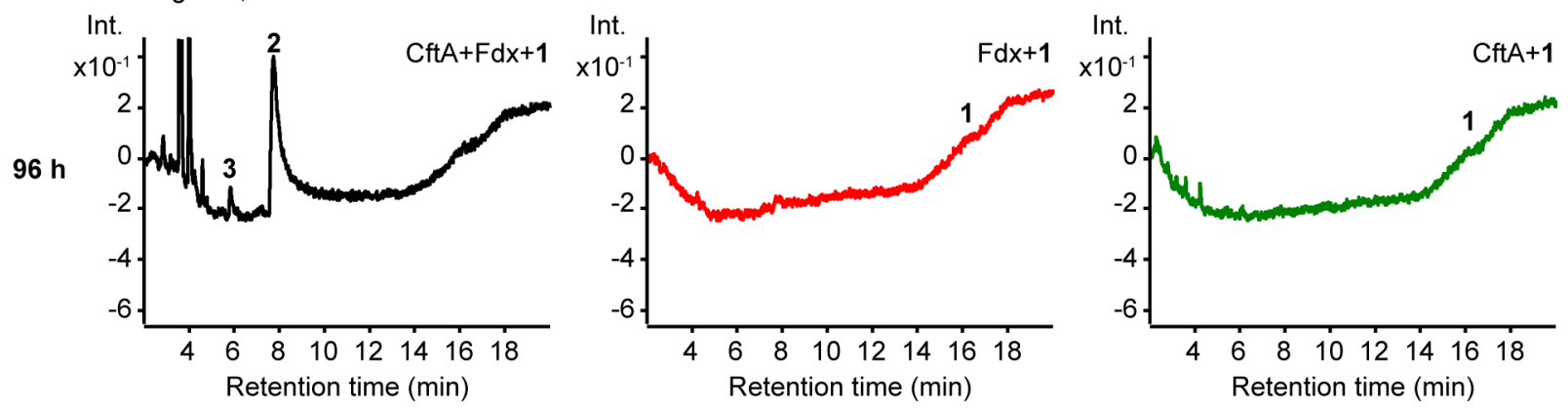

Figure S11. Total ion chromatograms (TICs, obtained by using precursor ion scan mode with fragmentation into daughter $[\mathrm{M}+\mathrm{H}]^{+}$ions with $m / z$ values of 139.2) and HPLC-UV/DAD chromatograms (detection at $320 \mathrm{~nm}$ ) generated from low resolution triple quadrupole HPLCMS/MS analyses of CftA reaction mixtures after $24 \mathrm{~h}$ (panel A) and $96 \mathrm{~h}$ (panel B) using ikarugamycin (1) as substrate. Ferredoxin/ferredoxin reductase redox system was abbreviated as $\mathrm{Fdx}$. Control reactions were performed without $\mathrm{CftA}$ or Fdx redox system. 
A.
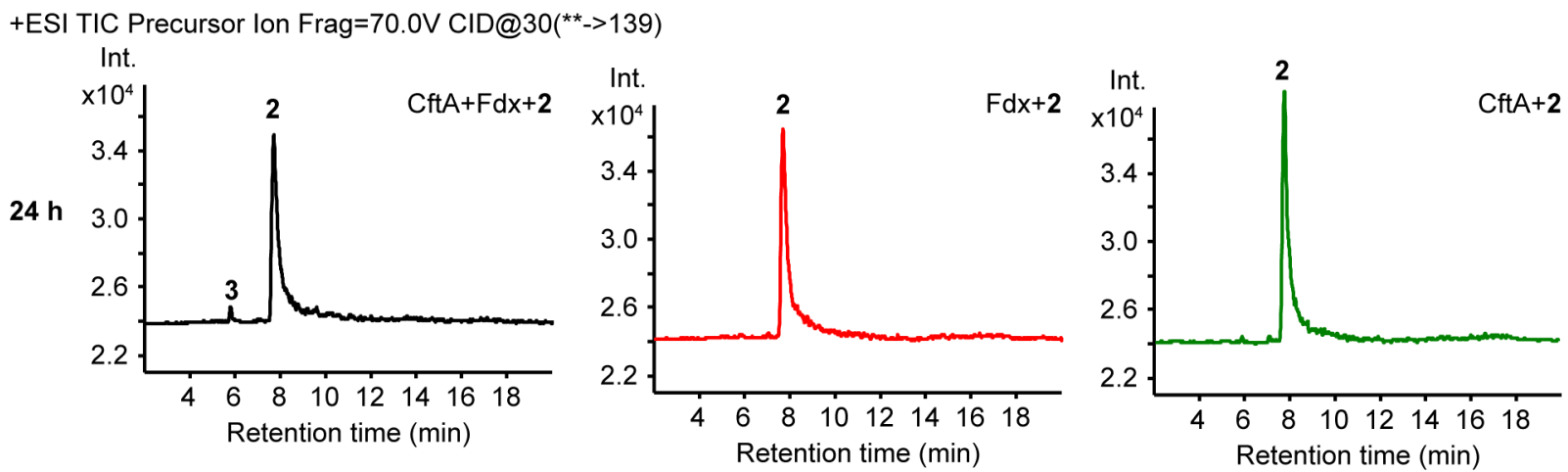

DAD1 - D: Sig:320, 8.0
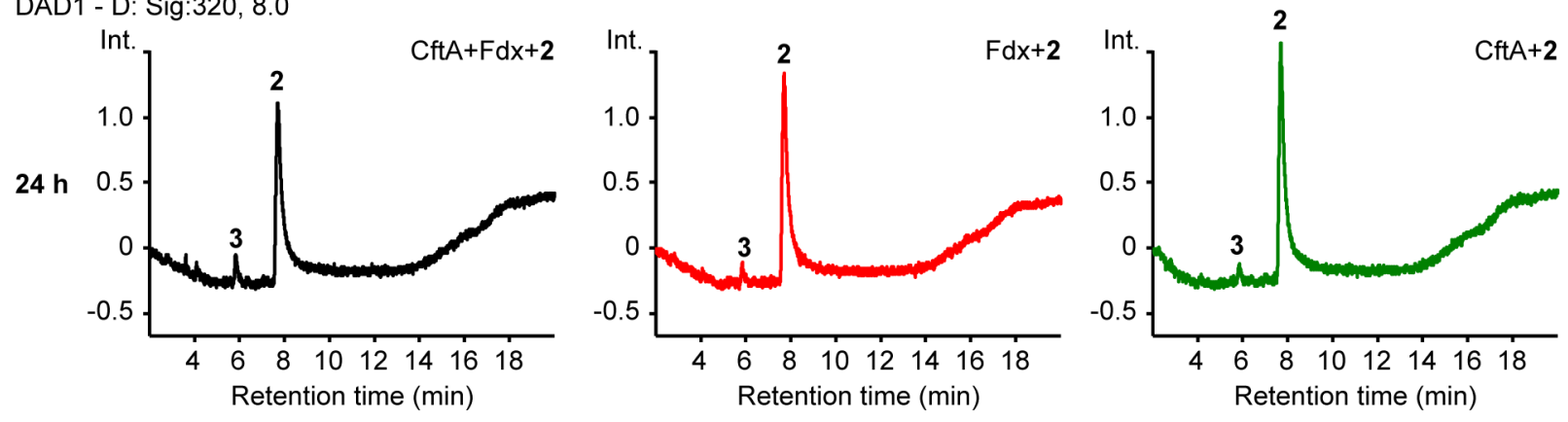

B.
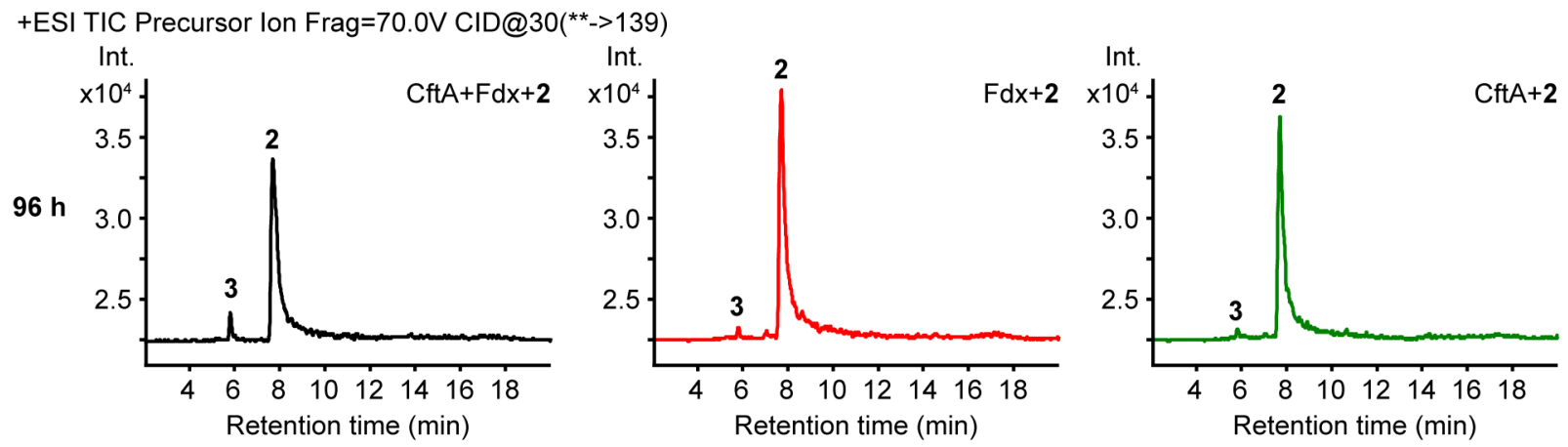

DAD1 - D: Sig:320, 8.0
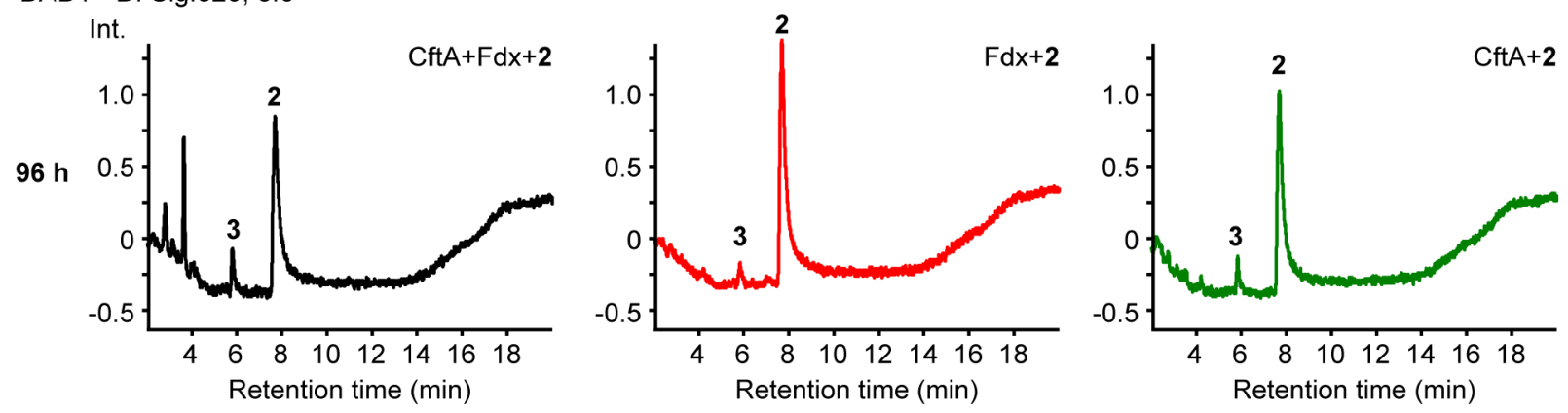

Figure S12. Total ion chromatograms (TICs, obtained by using precursor ion scan mode with fragmentation into daughter $[\mathrm{M}+\mathrm{H}]^{+}$ions with $m / z$ values of 139.2) and HPLC-UV/DAD chromatograms (detection at $320 \mathrm{~nm}$ ) generated from low resolution triple quadrupole HPLCMS/MS analyses of CftA reaction mixtures after $24 \mathrm{~h}$ (panel A) and $96 \mathrm{~h}$ (panel B) using clifednamide A (2) as substrate. Ferredoxin/ferredoxin reductase redox system was abbreviated as $\mathrm{Fdx}$. Control reactions were performed without CftA or Fdx redox system. 
A.

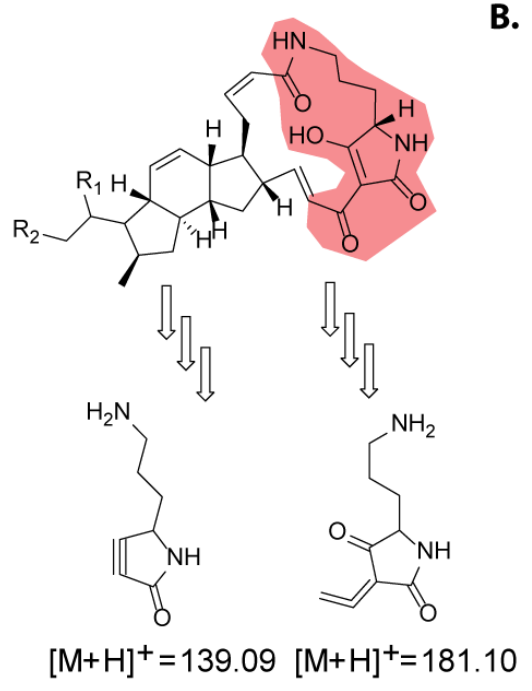

B.

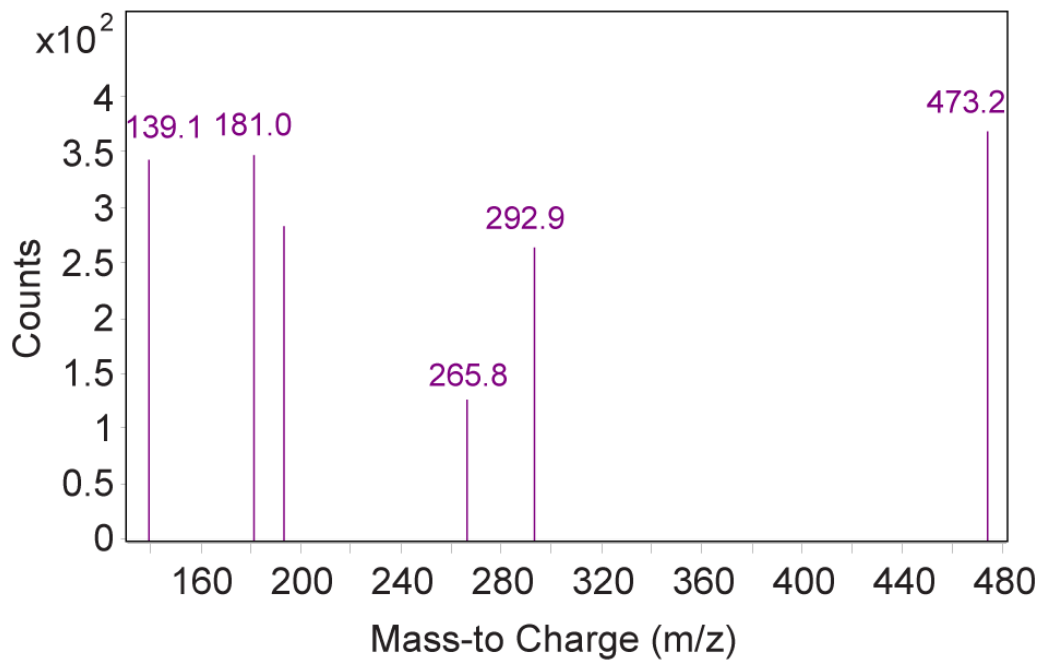

Figure S13. (a) Partial fragmentation indicating common daughter ions for clifednamide A/C. (b) $\mathrm{MS} / \mathrm{MS}$ analysis of the unknown clifednamide $\mathrm{C}$ isomer.

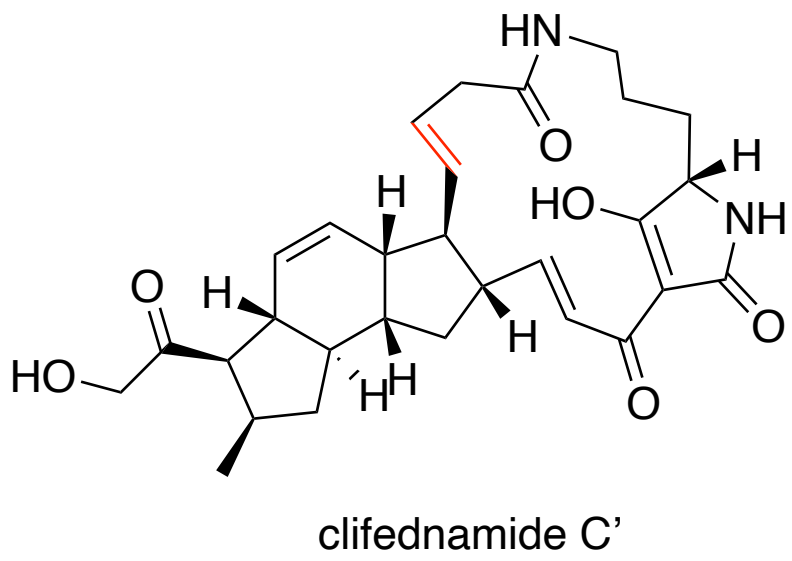

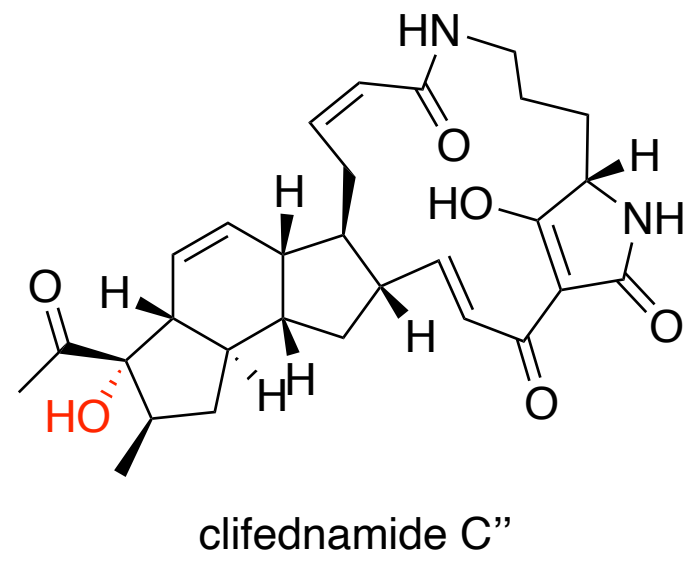

Figure S14. Potential isomeric structures of clifednamide $\mathrm{C}$ generated during in vitro conversion of ikarugamycin to clifednamides $\mathrm{A}$ and $\mathrm{C}$ (see main text Figure 4 and associated discussion). 
Reference sequence (1): MycG

Identities normalised by aligned length.

Colored by: identity

$\begin{array}{rrrr} & & \text { cov } & \text { pid } \\ 1 \text { MycG } & 100.0 \% & 100.0 \% \\ 2 \text { CbmC } & 99.5 \% & 45.6 \% \\ 3 \text { CftA } & 99.2 \% & 48.9 \% \\ 4 & \text { IkaD } & 99.2 \% & 45.0 \%\end{array}$

cov pid

1 MycG 100.0\% 100.0\%

2 CbmC $99.5 \% \quad 45.6 \%$

3 CftA $99.2 \% \quad 48.9 \%$

4 IkaD $99.2 \% \quad 45.0 \%$
1

$$
--
$$
-MTSAEPRAYPFNDVHGLTLAGRYGELQETEPVSRVRPPYGEEAWLVTRYEDVRAVLGDGRFVRGPSI 作 - - - - - - - - - MSDQHPPLPYPFAPR-GLDLDPTYAELRDR-APARIRMPYGDDAWLVTRYEDVRTVLADPRFSLAASM MPGQQEQQAPSEHPEQQELLTFPFPST-GLEFPPVYHELYQQ-RLTKVRLPYGDDAYLAIRYADVKTVLSDSRFSIVASL

$$
\alpha A \quad \beta 1 \quad \beta 2 \quad \alpha B \quad \beta 3
$$

81

TRDEPRTRPEMV-KGGLLSMDPPEHSRLRRLVVKAFTARRAESLRPRAREIAHELVDQMAATGQPADLVAMFARQLPVRV ERDQPRMRELSPGGDGLLSTDPPVHTRLRGVVARHFSARRVEQMRVVVREAAEGLLDRLEESGPPGDLVQDFAVPLSIAM GRDQPRMRPVARTGAGLFSTEPPDHTRLRSLVARQFSARRVEPLRARAGELADELIDGMVAAGQPADLVEDFAIPMPTTI GQDQPRTRAGARVGNGLFSLDPPQHSRLRSVLGRDFTPRRVEKLRERVRELTDQCLDRMEAAGSPADLVAHLAVPMPTAV

$\alpha C$

$\alpha \mathrm{D}$

B4

$\alpha \mathrm{E}$

$\begin{array}{rrr}\text { cov } & \text { pid } \\ 1 \text { MycG } 100.0 \% & 100.0 \%\end{array}$

2 CbmC $99.5 \% \quad 45.6 \%$

3 CftA $99.2 \% \quad 48.9 \%$

4 IkaD $99.2 \% \quad 45.0 \%$

ICELLGVPSADHDRFTRWSGAFLSTAEVTAEEMQEAAEQAYAYMGDLIDRRRKEPTDDLVSALVQARDQQDSLSEQELLD ICDLLGVPATDRHRVQKWLEVLLAQV-VTNEDLAAESGAYFAYLTGLVEERRREPGDDLLTCLVRAHYEEGRLSYDELIK ICEVLGIPAKDHRMLWHWAETVLSAI-TPQEVLATEGRAFMEYMVGVLELRGREPGDDLLTTLVRACREEGLISEEELLS VCEMMGVPEPDHHLFWGWAETILSND-TTPDDLIRRYQEFTAYMGAMVEERRARPTDDMFGMLVRACDEEGRITEIEMHA

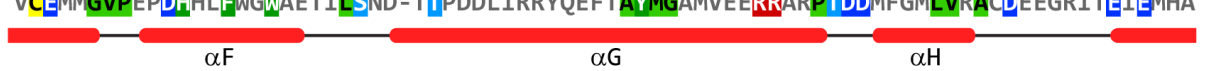

160

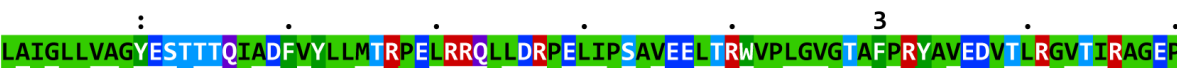
LSIELLTGGFVTTSKQIPNFCYLLLRRPEWLSRLREHPADLPVAIEEMLRYVPMPNGLVFCRYATEDVEVGGDLVRAGEP IACDLLIAGFVSTTNQIGNFFHQLLVHPTELTRLRERPELIPKAVEELMRYVPLLTGFNLARYATADVELGGITIRAGEA LASDLLSAGFVSTAHQIANFTAMLLARPERLQPLVDKPEQIPAAVEELMRHVPILSGFSFPRYATEDLELGGVTVRRGEA

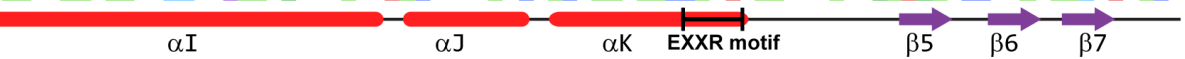

1 MycG $100.0 \% 100.0 \%$

2 CbmC $99.5 \% \quad 45.6 \%$

3 CftA $99.2 \% \quad 48.9 \%$

4 IkaD $99.2 \% \quad 45.0 \%$
:

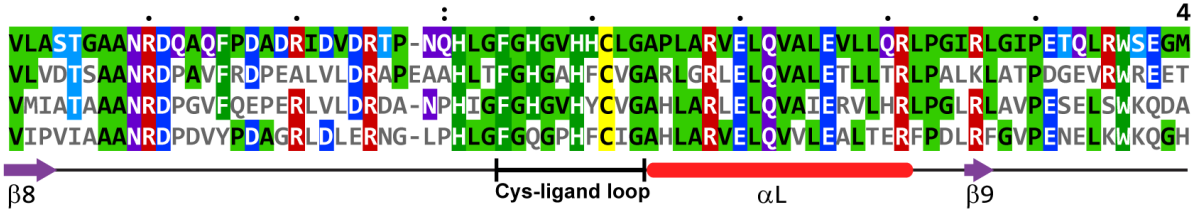

4400

Cov pid 321

$100.0 \% \quad 100.0 \%$

2 CbmC $99.5 \% \quad 45.6 \%$

3 CftA $99.2 \% \quad 48.9 \%$

4 IkaD 99.2\% $45.0 \%$

$\beta 8$

cov pid 401

1 MycG 100.0\% 100.0\%

$2 \mathrm{CbmC} 99.5 \% \quad 45.6 \%$

3 CftA $99.2 \% \quad 48.9 \%$

4 IkaD $99.2 \% \quad 45.0 \%$

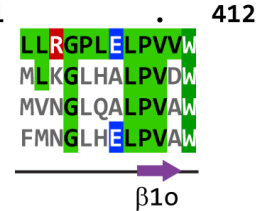

Figure S15. Primary protein sequence alignment by ClustalW for selected P450 enzymes - MycG (anchor), CbmC, CftA, and IkaD. ${ }^{5}$ Secondary structural motifs corresponding to primary sequence are provided below the alignment regions, where red rods represent $\alpha$-helices and purple arrows for $\beta$-turns. Sequence agreement is color coded by identity generated by M-view (PMID: 9632837) using the default built-in colormap for proteins. ${ }^{6}$ Only those residues that are identical to the reference sequence are colored. 
A.

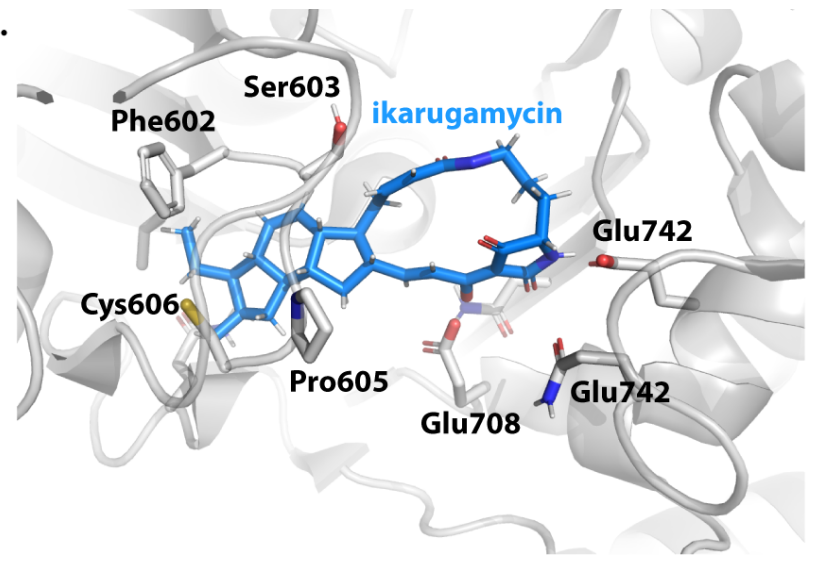

B.

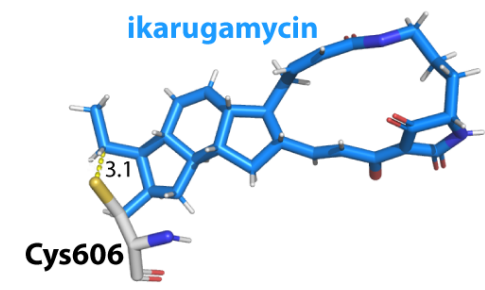

Figure S16. Molecular docking model of ikarugamycin in human hexokinase 2 (PDB: 5hfu). 


\section{Supporting Tables}

Table S1. MIBiG accession numbers of PTM biosynthetic gene clusters (BGCs) used in this paper.

\begin{tabular}{|c|c|c|c|c|c|c|c|}
\hline Compound & Strain & MIBiG & $\begin{array}{l}\text { PKS- } \\
\text { NRPS }\end{array}$ & $\mathbf{A D H}^{\mathbf{a}}$ & $\mathbf{F A S}^{\mathbf{b}}$ & $\begin{array}{c}\text { Oxido- } \\
\text { reductase }\end{array}$ & $\mathrm{CYPs}^{\mathrm{c}}$ \\
\hline ikarugamycin & $\begin{array}{c}\text { Streptomyces sp. } \\
\text { ZJ306 }\end{array}$ & BGC0001435 & IkaA & IkaC & N.A. & IkaB & N.A. \\
\hline clifednamide & $\begin{array}{c}\text { Streptomyces sp. } \\
\text { JV178 }\end{array}$ & BGC0001553 & $\mathrm{IkaA}^{\mathrm{d}}$ & IkaC & $\operatorname{Ftd}^{\mathrm{e}}$ & IkaB & $\mathrm{CftA}$ \\
\hline HSAF & $\begin{array}{l}\text { Lysobacter } \\
\text { enzymogenes }\end{array}$ & BGC0000999 & $\mathrm{IkaA}^{\mathrm{f}}$ & OX4 & $\operatorname{FtdA}^{g}$ & $\mathrm{OX} 1, \mathrm{OX} 2, \mathrm{OX} 3$ & N.A. \\
\hline pactamide & $\begin{array}{l}\text { Streptomyces } \\
\text { pactum }\end{array}$ & BGC0001726 & PtmA & $\mathrm{PbmC}$ & PtmD & PtmB1, PtmB2 & N.A. \\
\hline frontalamide & $\begin{array}{c}\text { Streptomyces sp. } \\
\text { SPB78 }\end{array}$ & BGC0000996 & FtdB & FtdE & FtdA & FtdC, FtdD & $\mathrm{FtdF}$ \\
\hline combamide & Streptomyces sp. & BGC0001556 & $\mathrm{CbmA}$ & N.A. & CbmE & $\mathrm{CbmB}, \mathrm{CbmC}$ & CbmD \\
\hline
\end{tabular}

${ }^{\mathrm{a} A D H}$, alcohol dehydrogenase. ${ }^{\mathrm{b}} \mathrm{FAS}$, fatty acid synthase. ${ }^{\mathrm{c}} \mathrm{CYPs}$, cytochrome P450. ${ }^{\mathrm{d} I k a A}$ homolog in clifednamide BGC. ${ }^{~}$ FtdA homolog in clifednamide BGC. ${ }^{\mathrm{f}}$ IkaA homolog in HSAF BGC. ${ }^{9} \mathrm{FtdA}$ homolog in HSAF BGC.

Table S2. Percent amino acid sequence identity of PKS-NRPS module in PTM BGCs.

\begin{tabular}{|c|c|c|c|c|c|c|}
\hline & FtdB & $\operatorname{PtmA}$ & IkaA & IkaA $^{a}$ & IkaA $^{b}$ & CbmA \\
\hline FtdB & 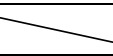 & 65 & 63.79 & 60.66 & 63.72 & 64.24 \\
\hline PtmA & 65.00 & $P^{2}$ & 66.07 & 62.26 & 66.7 & 67.1 \\
\hline IkaA & 63.79 & 66.07 & ${ }^{2}$ & 66.56 & 69.42 & 69.89 \\
\hline IkaA $^{\mathbf{a}}$ & 60.66 & 62.26 & 66.56 & 7 & 65.12 & 67.52 \\
\hline IkaA $^{b}$ & 63.72 & 66.7 & 69.42 & 65.1 & - & 68.84 \\
\hline CbmA & 64.24 & 67.1 & 69.89 & 67.52 & 68.84 & \\
\hline
\end{tabular}

${ }^{a}$ IkaA homolog in HSAF BGC. ${ }^{6} \mathrm{IkaA}$ homolog in clifednamide BGC.

Table S3. Percent amino acid sequence identity of alcohol dehydrogenase in PTM BGCs.

\begin{tabular}{|c|c|c|c|c|c|}
\hline & IkaC & IkaC $^{\text {a }}$ & PtmC & OX4 & FtdE \\
\hline IkaC & & 66.95 & 65.53 & 62.96 & 62.11 \\
\hline IkaC $^{\text {a }}$ & 66.95 & & 69.8 & 66.1 & 68.95 \\
\hline PtmC & 65.53 & 69.8 & & 68.09 & 68.38 \\
\hline OX4 & 62.96 & 66.1 & 68.09 & & 68.38 \\
\hline FtdE & 62.11 & 68.95 & 68.38 & 68.38 & \\
\hline
\end{tabular}

a IkaC homolog in clifednamide BGC.

Table S4. Percent amino acid sequence identity of fatty acid deasturase in PTM BGCs.

\begin{tabular}{|c|c|c|c|c|c|}
\hline & PtmD & FtdA $^{\mathbf{a}}$ & FtdA & FtdA $^{\mathbf{b}}$ & CbmE \\
\hline PtmD & & 50.83 & 53.42 & 56.14 & 55.6 \\
\hline FtdA $^{\mathbf{a}}$ & 50.83 & & 51.59 & 58.86 & 55.6 \\
\hline FtdA & 53.42 & 51.59 & & 60.74 & 59.57 \\
\hline FtdA $^{\mathbf{b}}$ & 56.14 & 58.86 & 60.74 & & 62.45 \\
\hline CbmE & 55.6 & 55.6 & 59.57 & 62.45 & \\
\hline
\end{tabular}

${ }^{a}$ FtdA homolog in HSAF BGC. ${ }^{b}$ FtdA homolog in clifednamide BGC. 
Table S5. List of strains and plasmids used in this study.

\begin{tabular}{cccc}
\hline Strain & Plasmid & Inducible Gene/Marker & Origin/Reference \\
\hline Streptomyces sp. strain NRRL F- 2890 & attBФC31::pED2 & Clifednamide producer & PMID: 29249153 \\
JV772 & None & Protein expression strain & Agilent \\
E. coli BL21(DE3) coli BL21(DE3) & pET28a & cftA & This work \\
Bacillus subtilis ATCC 6633 & None & None & ATCC \\
Staphylococcus aureus ATCC 11632 & None & None & ATCC \\
Micrococcus luteus ATCC 11632 & None & None & ATCC \\
Candida albicans ATCC 48130 & None & None & ATCC \\
\hline
\end{tabular}

Table S6. Nucleotide sequence of codon-optimized cftA.

\begin{tabular}{|c|c|}
\hline Gene & Codon optimized nucleotide Sequence \\
\hline cftA & $\begin{array}{l}\text { CATATGCACATGCACATGAGCGACCAACACCCGCCGCTGCCGTACCCGTTTGCGCCGCGTGGCCTGG } \\
\text { ACCTGGACCCGACCTATGCGGAACTGCGTGATCGTGCGCCGGCGCGTATTCGTATGCCGTACGGCG } \\
\text { ACGATGCGTGGCTGGTGACCCGTTATGAGGATGTGCGTACCGTGCTGGCGGACCCGCGTTTCAGCCT } \\
\text { GGCGGCGAGCATGGGTCGTGACCAGCCGCGTATGCGTCCGGTTGCGCGTACCGGTGCGGGCCTGTT } \\
\text { CAGCACCGAACCGCCGGATCACACCCGTCTGCGTAGCCTGGTGGCGCGTCAGTTTAGCGCGCGTCGT } \\
\text { GTTGAACCGCTGCGTGCGCGTGCGGGTGAACTGGCGGACGAGCTGATTGATGGTATGGTGGCGGCG } \\
\text { GGTCAACCGGCGGACCTGGTTGAAGATTTCGCGATCCCGATGCCGACCACCATCATTTCGAGGTGC } \\
\text { TGGGTATTCCGGCGAAGGACCACCGTATGCTGTGGCACTGGGCGGAAACCGTGCTGAGCGCGATCA } \\
\text { CCCCGCAAGAAGTTCTGGCGACCGAGGGCCGTGCGTTTATGGAGTACATGGTGGGTGTTCTGGAAC } \\
\text { TGCGTGGCCGTGAGCCGGGTGACGATCTGCTGACCACCCTGGTTCGTGCGTGCCGTGAGGAAGGCC } \\
\text { TGATCAGCGAGGAAGAGCTGCTGAGCATCGCGTGCGATCTGCTGATTGCGGGCTTCGTGAGCACCA } \\
\text { CCAACCAGATTGGTAACTTCTTTCACCAACTGCTGGTTCACCCGACCGAACTGACCCGTCTGCGTGA } \\
\text { ACGTCCGGAGCTGATCCCGAAAGCGGTGGAAGAGCTGATGCGTTACGTTCCGCTGCTGACCGGTTTT } \\
\text { AACCTGGCGCGTTATGCGACCGCGGACGTGGAACTGGGTGGCATCACCATTCGTGCGGGCGAGGCG } \\
\text { GTTATGATTGCGACCGCTGCGGCGAACCGTGATCCGGGTGTGTTCCAGGAACCGGAGCGTCTGGTTC } \\
\text { TGGACCGTGATGCGAACCCGCACATCGGTTTTGGCCACGGTGTGCACTATTGCGTTGGCGCGCACCT } \\
\text { GGCGCGTCTGGAACTGCAAGTGGCGATTGAGCGTGTTCTGCACCGTCTGCCGGGTCTGCGTCTGGCG } \\
\text { GTGCCGGAGAGCGAACTGAGCTGGAAACAAGATGCGATGGTGAACGGTCTGCAAGCGCTGCCGGT } \\
\text { GGCGTGGTAGAAGCTT }\end{array}$ \\
\hline
\end{tabular}

Table S7. Protein sequence of $N$-His6-tagged CftA.

\begin{tabular}{|l|l|}
\hline Gene & Primary Protein Sequences of $\boldsymbol{N}$-His 6 -CftA (his tag highlighted) \\
\hline$c f t A$ & MGSSHHHHHHSSGLVPRGSHMHMSDQHPPLPYPFAPRGLDLDPTYAELRDRAPARIRMPYGDDAWLVT \\
& RYEDVRTVLADPRFSLAASMGRDQPRMRPVARTGAGLFSTEPPDHTRLRSLVARQFSARRVEPLRARAG \\
& ELADELIDGMVAAGQPADLVEDFAIPMPTTIICEVLGIPAKDHRMLWHWAETVLSAITPQEVLATEGRAF \\
& MEYMVGVLELRGREPGDDLLTTLVRACREEGLISEEELLSIACDLLIAGFVSTTNQIGNFFHQLLVHPTEL \\
& TRLRERPELIPKAVEELMRYVPLLTGFNLARYATADVELGGITIRAGEAVMIATAAANRDPGVFQEPERL \\
& VLDRDANPHIGFGHGVHYCVGAHARLELQVAIERVLHRLPGLRLAVPESELSWKQDAMVNGLQALPVA \\
& W \\
\hline
\end{tabular}


Table S8. Select P450 enzymes used for the phylogenetic analysis.

\begin{tabular}{|c|c|c|c|c|c|}
\hline P450 & Natural product & Strain & Function & Accession number & Reference \\
\hline AmphL & Amphotericin & Streptomyces nodosus & Hydroxylation & AAK73504.1 & PMID: 11451671 \\
\hline AziB 1 & Azinomycin & Arthrobacter sp. 162MFSha1.1 & Hydroxylation & WP_018771011.1 & PMID: 20485749 \\
\hline $\mathrm{CbmC}$ & Combamide & Streptomyces sp. & Hydroxylation/oxidation to ketone & AWO77090.1 & PMID: 29792712 \\
\hline $\mathrm{CftA}$ & Clifednamide & Streptomyces sp. JV178 & Hydroxylation/oxidation to ketone & AUD08664.1 & PMID: 29249153 \\
\hline ChmPI & Chalcomycin & Streptomyces bikiniensis & Hydroxylation & AAS79447.1 & PMID: 15561847 \\
\hline CYP170A1 & Albaflavenone & Streptomyces coelicolor & Hydroxylation/oxidation to ketone & Q9K498.1 & PMID: 18234666 \\
\hline DoxA & Doxorubicin & Streptomyces peucetius & Hydroxylation/oxidation to ketone & Q9ZAU3.1 & PMID: 9864343 \\
\hline EryF & Erythromycin & Saccharopolyspora erythraea & Hydroxylation & WP_016577953.1 & PMID: 9335291 \\
\hline EryK & Erythromycin & Saccharopolyspora erythraea & Hydroxylation & WP_009950895.1 & PMID: 7849045 \\
\hline Fma-P450 & Fumagillin & Aspergillus fumigatus & Hydroxylation/epoxidation & A0A067Z9B6.1 & PMID: 24568283 \\
\hline Fr9R & Spliceostatin & Burkholderia sp. FERM BP-3421 & Hydroxylation & AIC32704.1 & PMID: 25097259 \\
\hline GfsF & FD-891 & Streptomyces graminofaciens & Hydroxylation/epoxidation & BAJ16472.1 & PMID: 28869713 \\
\hline IkaD & Capsimycin & Streptomyces xiamenensis 318 & Hydroxylation & SXIM_40690 & PMID: 28098172 \\
\hline MeiE & Meilingmycin & Streptomyces nanchangensis & Hydroxylation & AAM97314.1 & PMID: 20348291 \\
\hline MycCI & Mycinamicin & Micromonospora griseorubida & Hydroxylation & BAC57023.2 & PMID: 22547618 \\
\hline MycG & Mycinamicin & Micromonospora griseorubida & Hydroxylation/epoxidation & Q59523.1 & PMID: 22547618 \\
\hline NcsB3 & Neocarzinostatin & $\begin{array}{c}\text { Streptomyces carzinostaticus subsp. } \\
\text { neocarzinostaticus }\end{array}$ & Hydroxylation & AAM77997.1 & PMID: 20735485 \\
\hline NysL & Nystatin & Streptomyces noursei ATCC 11455 & Hydroxylation & AAF71769.1 & PMID: 16597951 \\
\hline
\end{tabular}




\begin{tabular}{|c|c|c|c|c|c|}
\hline OxyD & $\beta$-hydroxytyrosine & Amycolatopsis orientalis & Hydroxylation & CCD33151.1 & PMID: 20519494 \\
\hline PenD & Pentalenolactone & Streptomyces exfoliatus & Epoxidation & ADO85592.1 & PMID: 21284395 \\
\hline PikC & Narbomycin & Streptomyces venezuelae & Hydroxylation & AAC64105.1 & PMID: 9831532 \\
\hline Pl-p450-1 & Pleuromutilin & Pleurotus sapidus & Hydroxylation & CAL69751 & PMID: 27143514 \\
\hline Pl-p450-3 & Pleuromutilin & Pleurotus sapidus & Hydroxylation & CAJ00405 & PMID: 27143514 \\
\hline PldB & Pladienolide & Streptomyces platensis & Hydroxylation & ВАН02272.1 & PMID: 18997414 \\
\hline PntD & Pentalenolactone & Streptomyces arenae & Epoxidation & ADO85576.1 & PMID: 21284395 \\
\hline TamI & Tirandamycin & Streptomyces sp. 307-9 & Hydroxylation/epoxidation & ADC79647.1 & PMID: 33569241 \\
\hline TstR & Thailanstatins & Burkholderia thailandensis & Epoxidation & AGN11891.1 & PMID: 23517093 \\
\hline TxtC & Thaxtomin & Streptomyces acidiscabies & Hydroxylation & AAL36838.1 & PMID: 30604585 \\
\hline TyII & Tylosin & Streptomyces fradiae & Hydroxylation & AAA21341.1 & PMID: 7984112 \\
\hline TylHI & Tylosin & Streptomyces fradiae & Hydroxylation & AAD41818.1 & PMID: 31488542 \\
\hline ZbmVIIc & Zorbamycin & Streptomyces flavoviridis & Hydroxylation & ACG60779.1 & PMID: 19081934 \\
\hline
\end{tabular}


Table S9. Percent amino acid sequence identity of selected P450 enzymes.

\begin{tabular}{|c|c|c|c|c|}
\hline & CftA & CbmC & IkaD & MycG \\
\hline CftA & & 53.92 & 46.17 & 49.49 \\
\hline CbmC & 53.92 & & 44.39 & 45.57 \\
\hline IkaD & 46.17 & 44.39 & & 52.67 \\
\hline MycG & 49.49 & 45.57 & 52.67 & \\
\hline
\end{tabular}

Table S10. Antimicrobial activity $\left(\mathrm{MIC}_{90}\right)$ of ikarugamycin and clifednamide A/C. ${ }^{\text {a }}$

\begin{tabular}{ccccc}
\hline Strain & $\begin{array}{c}\text { Ikarugamycin } \\
(\boldsymbol{\mu M})\end{array}$ & $\begin{array}{c}\text { Clifednamide A } \\
(\boldsymbol{\mu M})\end{array}$ & $\begin{array}{c}\text { Clifednamide C } \\
(\boldsymbol{\mu M})\end{array}$ & $\begin{array}{c}\text { Ampicillin } \\
(\boldsymbol{\mu} \mathbf{g} / \mathbf{m L})\end{array}$ \\
\hline B. subtilis ATCC 6633 & 45 & $>360$ & $>360$ & $<2.4$ \\
S. aureus ATCC 11632 & 22.5 & $>360$ & $>360$ & 19.5 \\
M. luteus ATCC 10240 & 22.5 & $>360$ & $>360$ & 9.8 \\
C. albicans ATCC 48130 & 22.5 & $>360$ & $>360$ & n.a. \\
\hline
\end{tabular}

${ }^{\mathrm{a}} \mathrm{MIC}_{90}$ values were determined in biological triplicates using the broth microdilution assay in MHII medium following CLSI guidelines.

Table S11. Calculated physiochemical properties of ikarugamycin and clifednamides A/C. ${ }^{\mathrm{a}}$

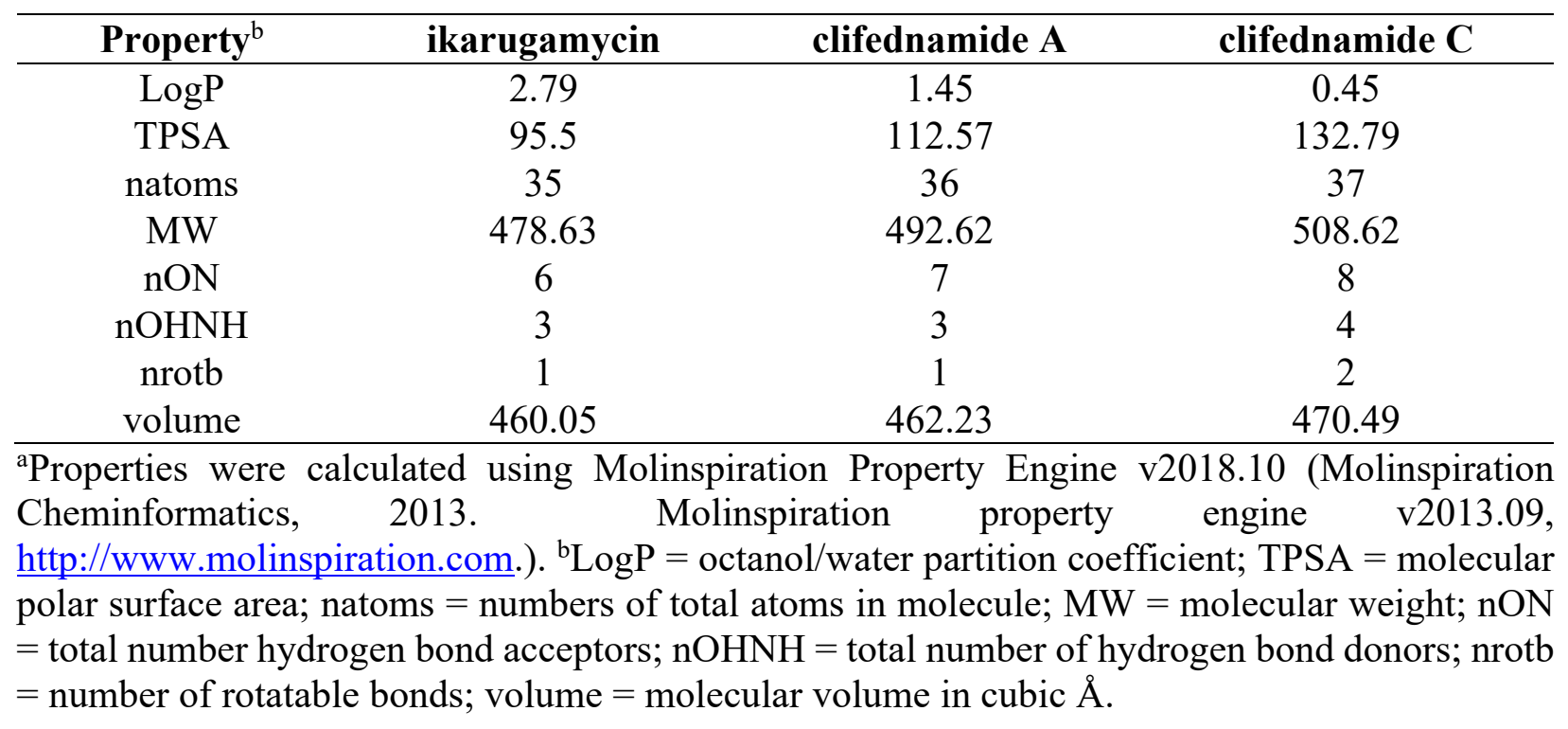




\section{Compound Characterization}

Table S12. NMR spectroscopic data for pure clifednamide C in DMSO- $d 6$ ( $\delta$ in ppm, $J$ in $\mathrm{Hz}$, $600 / 150 \mathrm{MHz}$ ).

\begin{tabular}{|c|c|c|c|c|c|}
\hline position & $\delta \mathrm{C}$ & type & $\delta \mathbf{H}$ & multiplicity & $J$ \\
\hline 1 & 165.5 & $\mathrm{C}$ & & & \\
\hline 2 & 124.6 & $\mathrm{CH}$ & 5.83 & $\mathrm{~d}$ & 11.4 \\
\hline 3 & 124.6 & $\mathrm{CH}$ & 5.94 & $\mathrm{dd}$ & $11.4,11.4$ \\
\hline 4 & 24.4 & $\mathrm{CH} 2$ & 2.11 & $\mathrm{~m}$ & \\
\hline 4 & 24.4 & $\mathrm{CH} 2$ & 3.42 & $\mathrm{~m}$ & \\
\hline 5 & 47.9 & $\mathrm{CH}$ & 1.32 & $\mathrm{~m}$ & \\
\hline 6 & 42.0 & $\mathrm{CH}$ & 2.50 & $\mathrm{~m}$ & \\
\hline 7 & 129.0 & $\mathrm{CH}$ & 5.63 & $\mathrm{~d} ?$ & 9.8 \\
\hline 8 & 129.0 & $\mathrm{CH}$ & 5.70 & $\mathrm{~d} ?$ & 9.8 \\
\hline 9 & 42.6 & $\mathrm{CH}$ & 2.28 & $\mathrm{~m}$ & \\
\hline 10 & 52.5 & $\mathrm{CH}$ & 2.79 & dd & $11.1,11.1$ \\
\hline 11 & 33.4 & $\mathrm{CH}$ & 2.57 & $\mathrm{~m}$ & \\
\hline 12 & 38.5 & $\mathrm{CH} 2$ & 0.71 & $\mathrm{~m}$ & \\
\hline 12 & 38.5 & $\mathrm{CH} 2$ & 2.11 & $\mathrm{~m}$ & \\
\hline 13 & 47.1 & $\mathrm{CH}$ & 1.19 & $\mathrm{~m}$ & \\
\hline 14 & 40.4 & $\mathrm{CH}$ & 2.05 & $\mathrm{~m}$ & \\
\hline 15 & 36.4 & $\mathrm{CH} 2$ & 1.12 & $\mathrm{~m}$ & \\
\hline 15 & 36.4 & $\mathrm{CH} 2$ & 2.04 & $\mathrm{~m}$ & \\
\hline 16 & 47.1 & $\mathrm{CH}$ & 2.27 & $\mathrm{~m}$ & \\
\hline 17 & 137.6 & $\mathrm{CH}$ & 5.95 & $\mathrm{~m}$ & \\
\hline 18 & 131.5 & $\mathrm{CH}$ & 7.49 & $\mathrm{~d}$ & 15.4 \\
\hline 19 & 181.2 & $\mathrm{C}$ & & & \\
\hline 20 & 103.6 & $\mathrm{C}$ & & & \\
\hline 21 & 176.7 & $\mathrm{C}$ & & & \\
\hline \multicolumn{6}{|l|}{22} \\
\hline 23 & 59.3 & $\mathrm{CH}$ & 4.03 & $\mathrm{~m}$ & \\
\hline 24 & 195.5 & $\mathrm{C}$ & & & \\
\hline 25 & 26.8 & $\mathrm{CH} 2$ & 1.67 & $\mathrm{~m}$ & \\
\hline 25 & 26.8 & $\mathrm{CH} 2$ & 1.73 & $\mathrm{~m}$ & \\
\hline 26 & 21.1 & $\mathrm{CH} 2$ & 1.16 & $\mathrm{~m}$ & \\
\hline 26 & 21.1 & $\mathrm{CH} 2$ & 1.26 & $\mathrm{~m}$ & \\
\hline 27 & 37.6 & $\mathrm{CH} 2$ & 2.40 & $\mathrm{~m}$ & \\
\hline 27 & 37.6 & $\mathrm{CH} 2$ & 3.29 & $\mathrm{~m}$ & \\
\hline \multicolumn{6}{|l|}{28} \\
\hline 29 & 212.3 & $\mathrm{C}$ & & & \\
\hline 30 & 68.5 & $\mathrm{CH} 2$ & 4.04 & $\mathrm{~d}$ & 18.7 \\
\hline 30 & 68.5 & $\mathrm{CH} 2$ & 4.10 & $d$ & 18.7 \\
\hline 31 & 18.6 & $\mathrm{CH} 3$ & 0.75 & $\mathrm{~d}$ & 6.9 \\
\hline
\end{tabular}




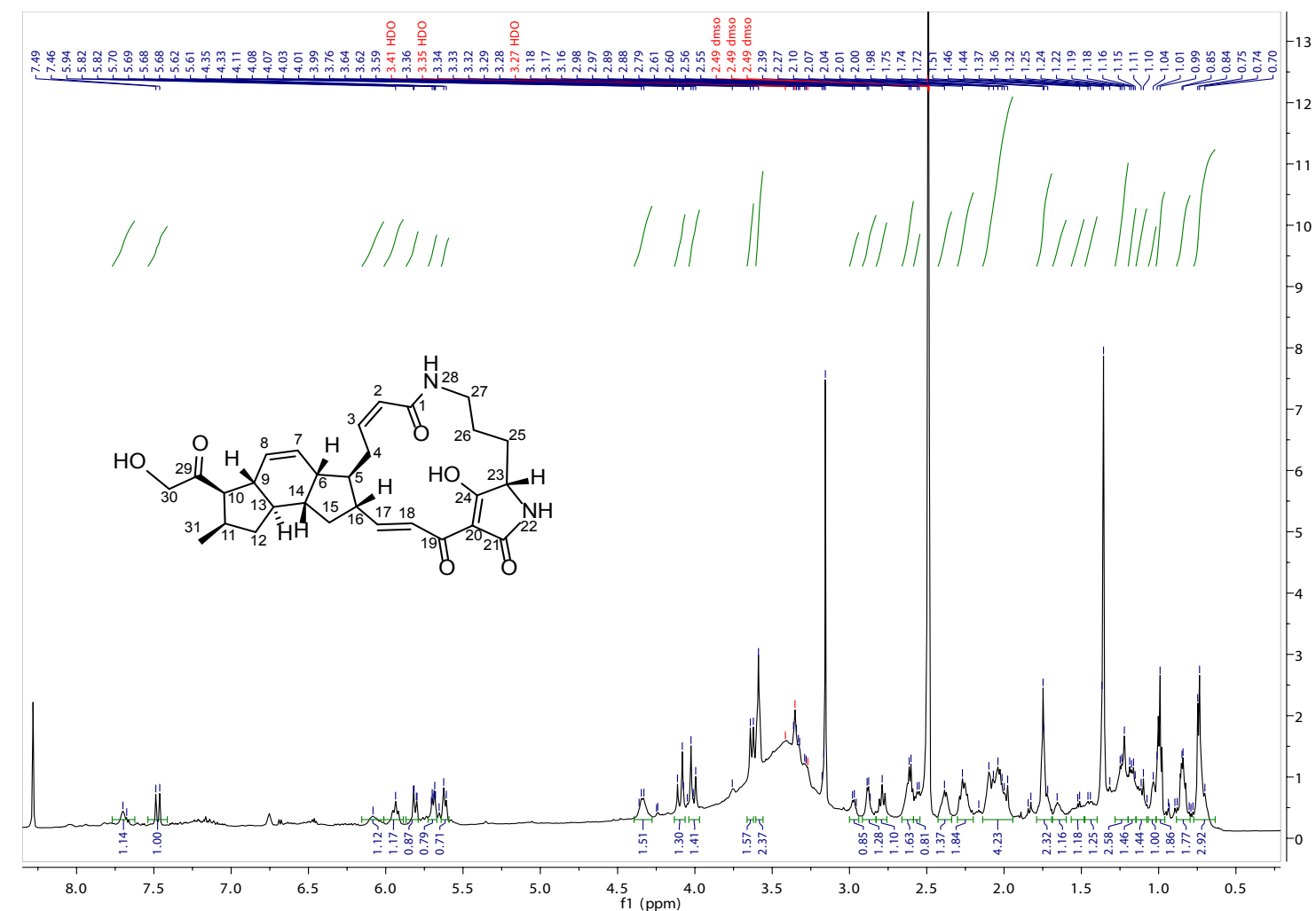

Figure S17. The ${ }^{1} \mathrm{H}-\mathrm{NMR}$ (DMSO- $d 6,600 \mathrm{MHz}$ ) spectrum of purified clifednamide C.

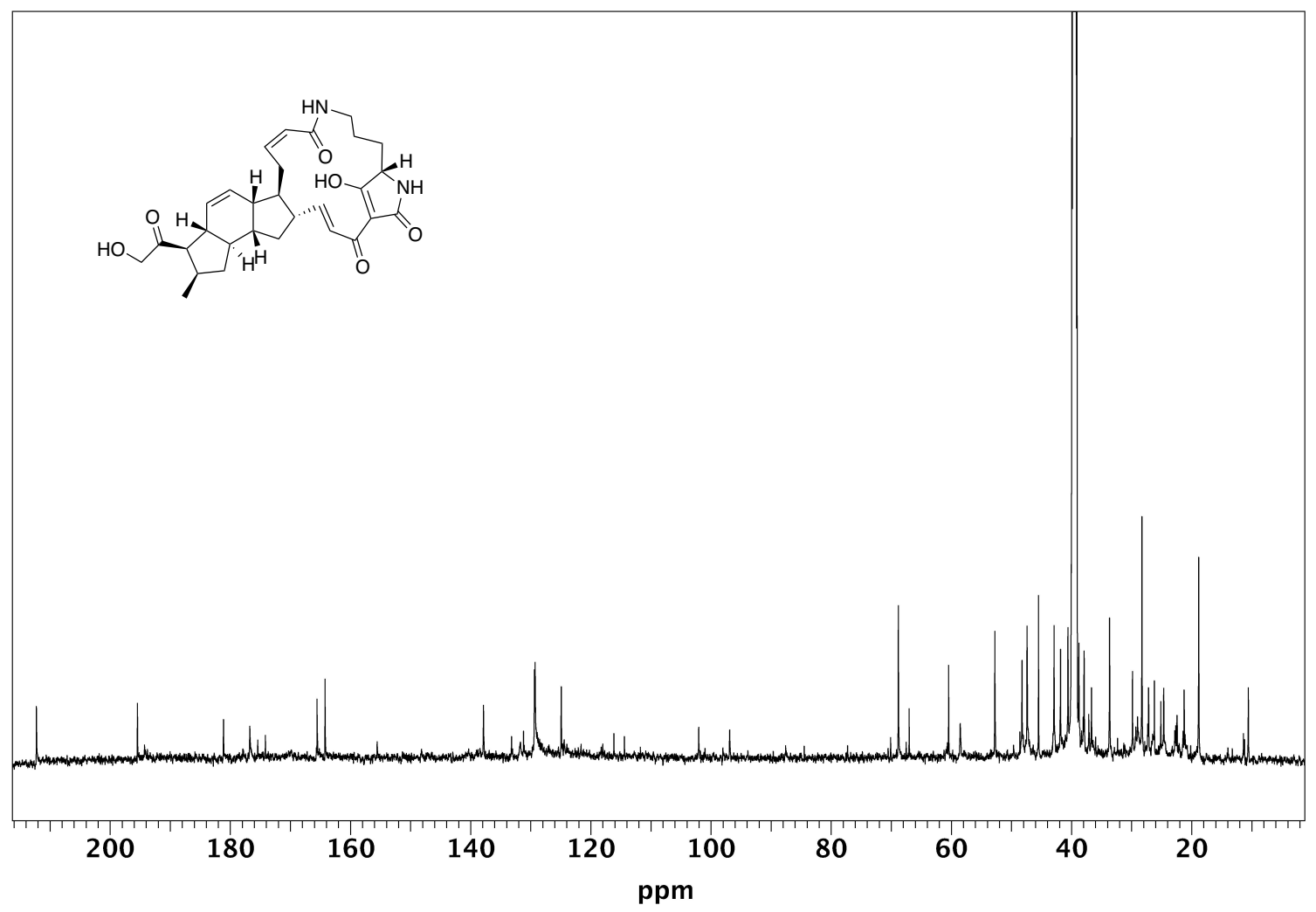

Figure S18. The ${ }^{13} \mathrm{C}-\mathrm{NMR}$ (DMSO- $d 6,150 \mathrm{MHz}$ ) spectrum of purified clifednamide C. 


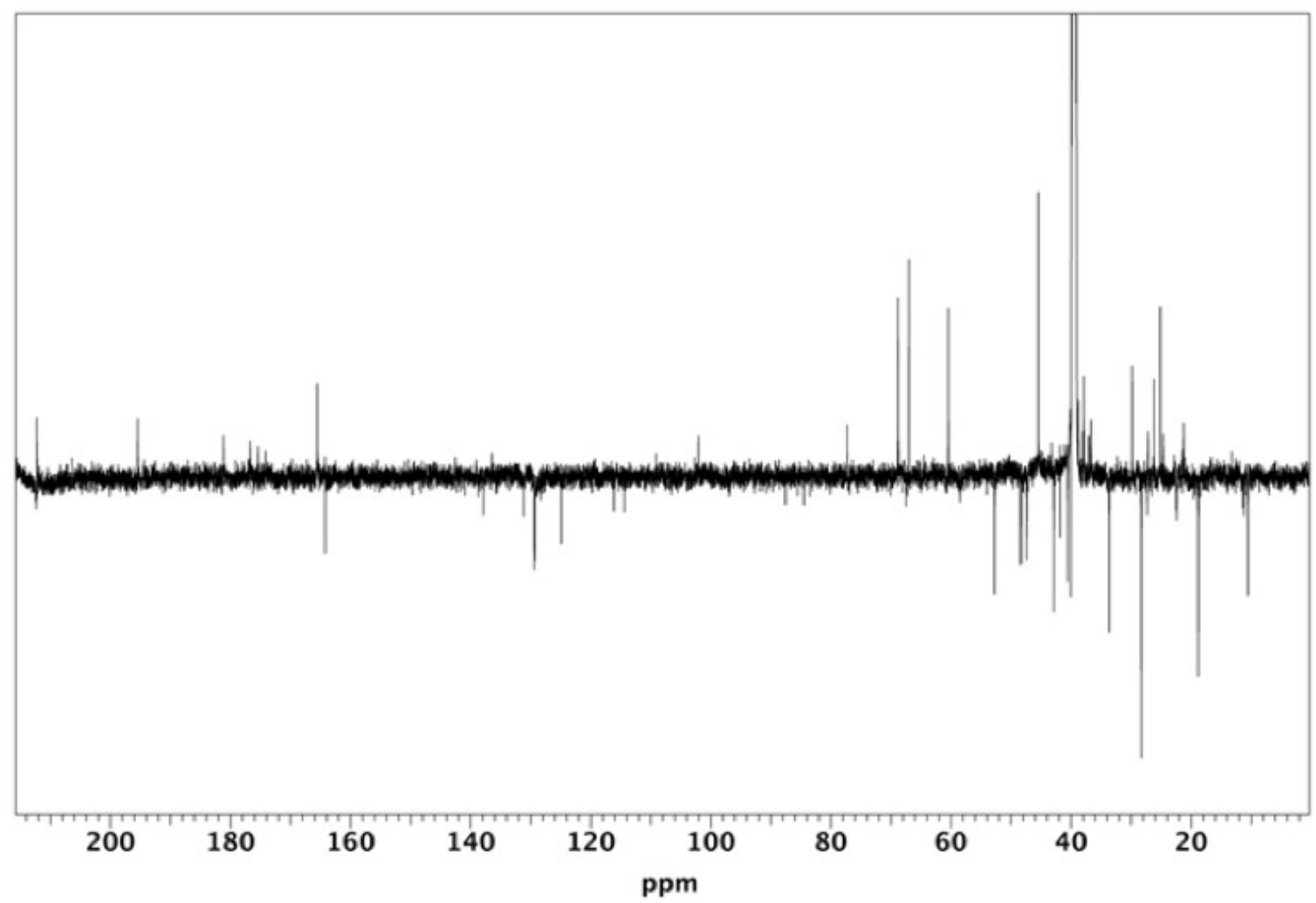

Figure S19. The APT (DMSO- $d 6,150 \mathrm{MHz}$ ) spectrum of purified clifednamide C.

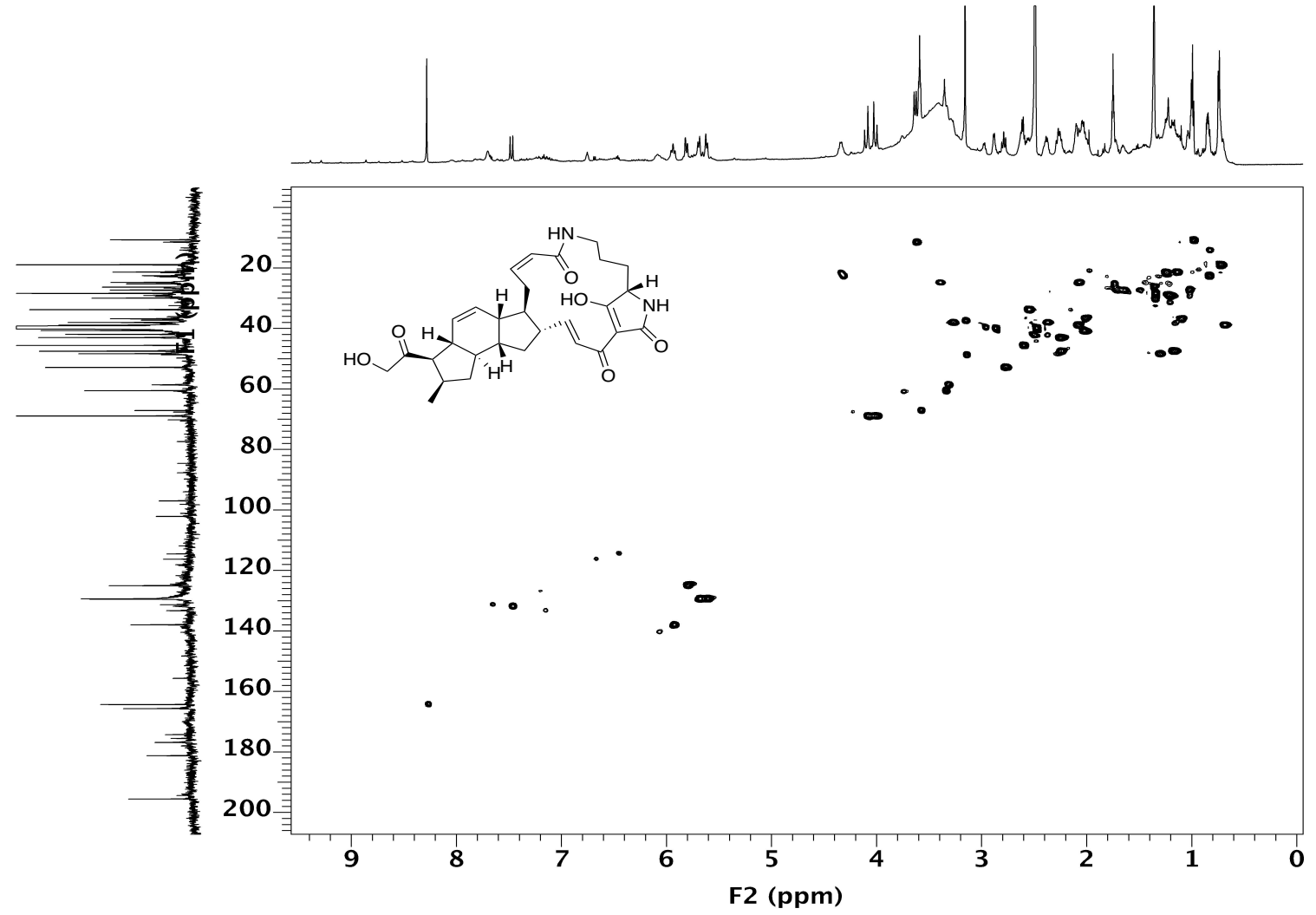

Figure S20. The HSQC (DMSO- $d 6,600 / 150 \mathrm{MHz}$ ) spectrum of purified clifednamide C. 


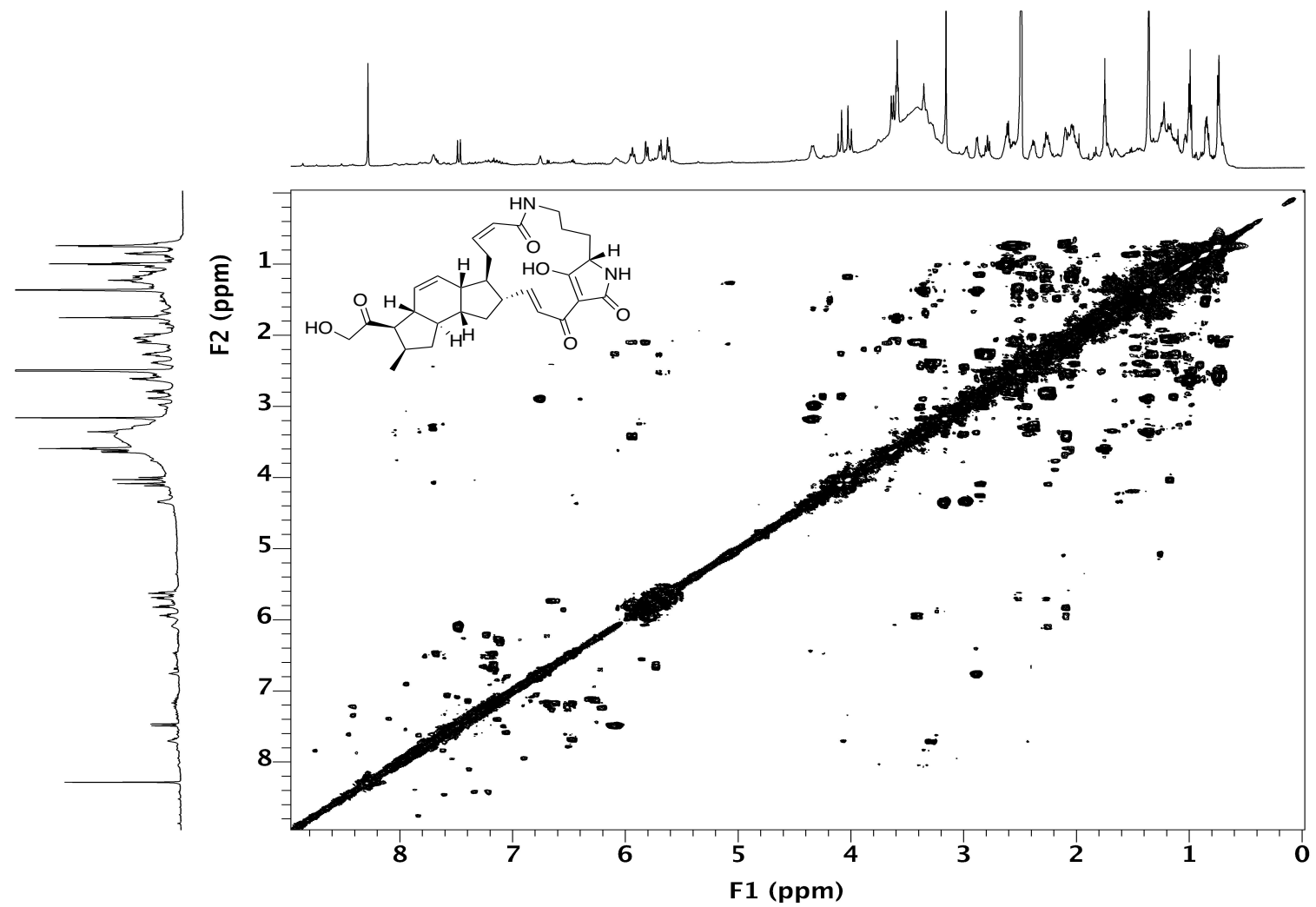

Figure S21. The COSY (DMSO- $d 6,600 \mathrm{MHz}$ ) spectrum of purified clifednamide C.

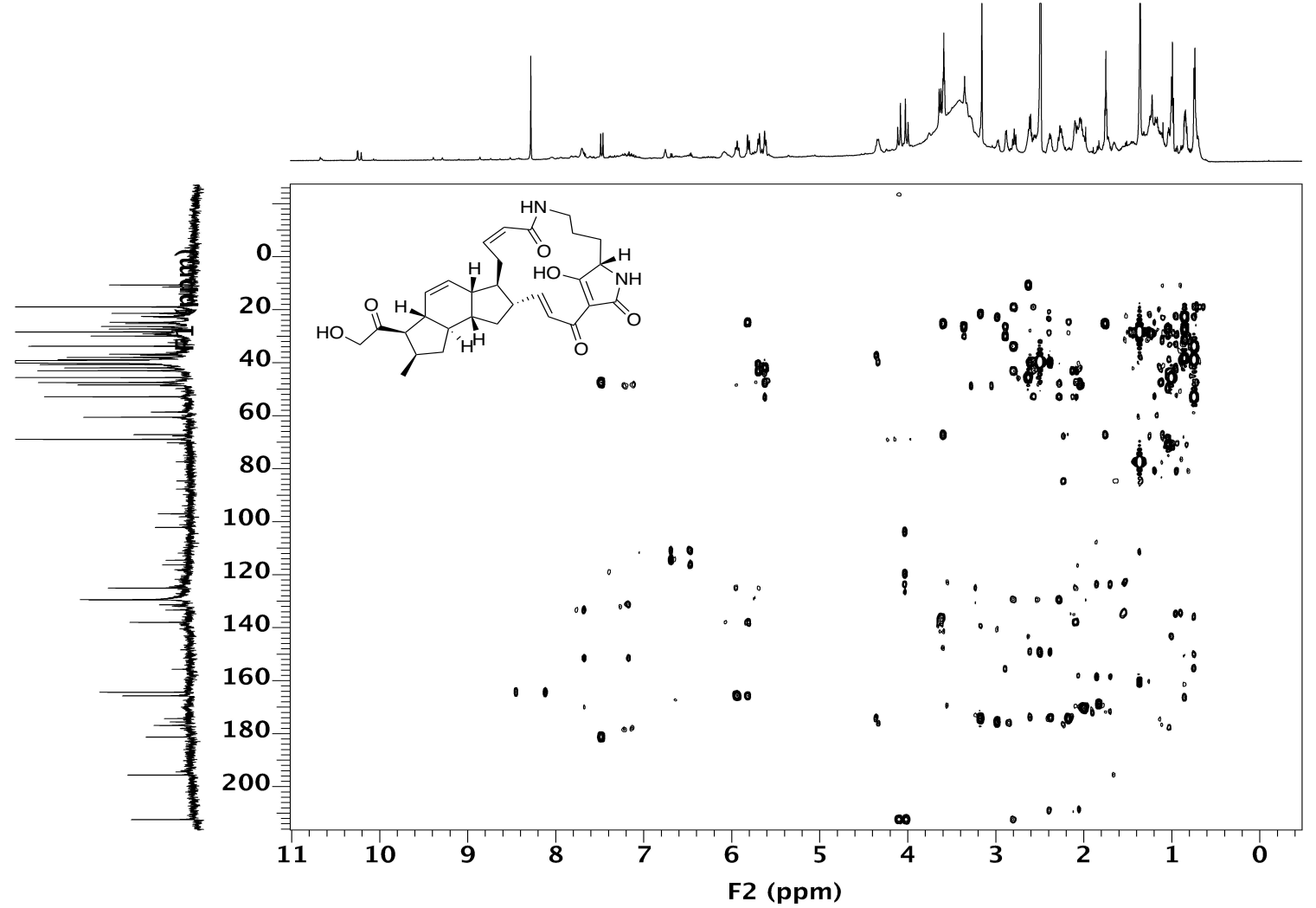

Figure S22. The HMBC (DMSO- $d 6,600 / 150 \mathrm{MHz}$ ) spectrum of purified clifednamide C. 


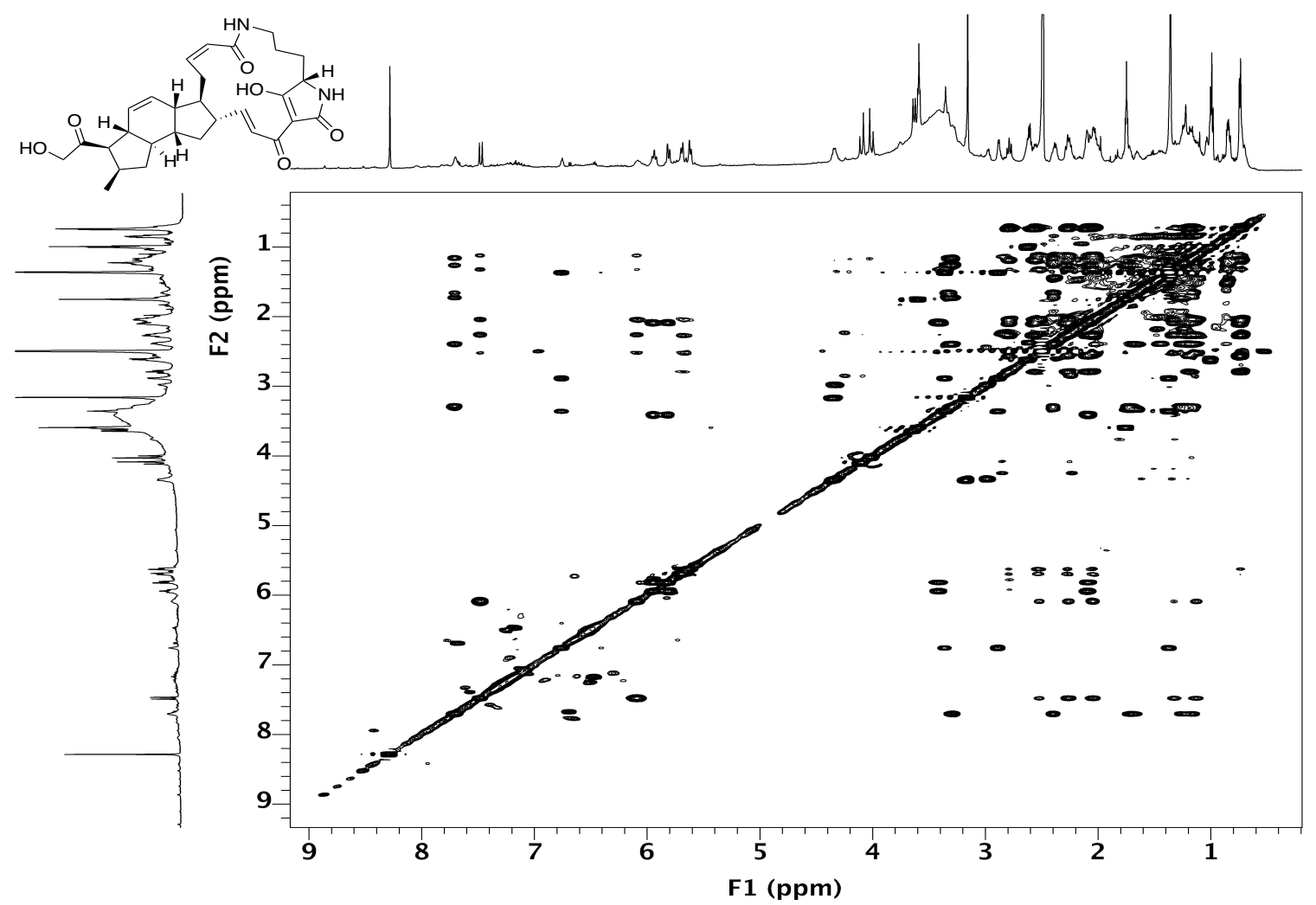

Figure S23. The TOCSY (DMSO-d6, $600 \mathrm{MHz}$ ) spectrum of purified clifednamide C.

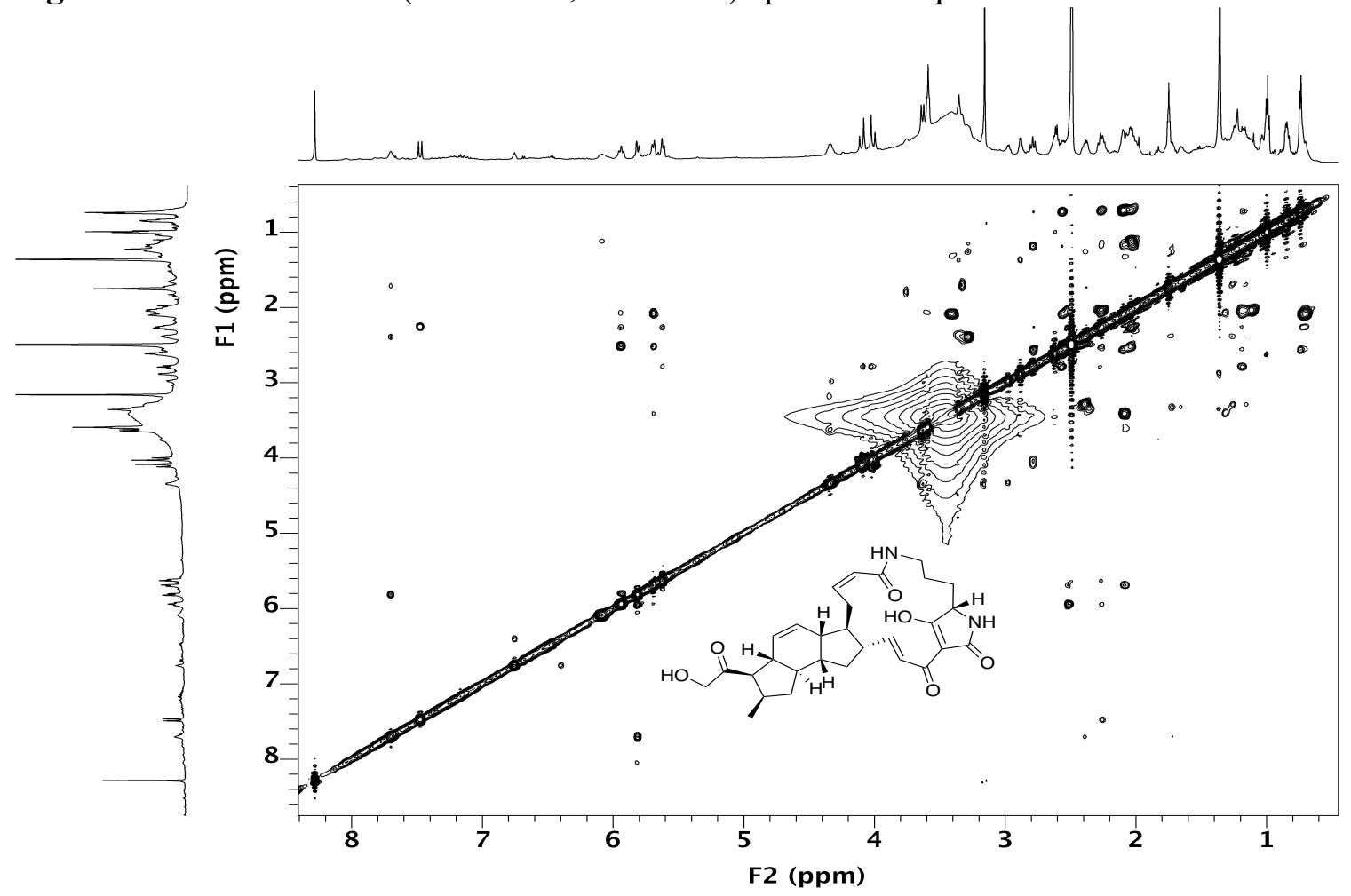

Figure S24. The NOESY (DMSO-d6, $600 \mathrm{MHz}$ ) spectrum of purified clifednamide C. 


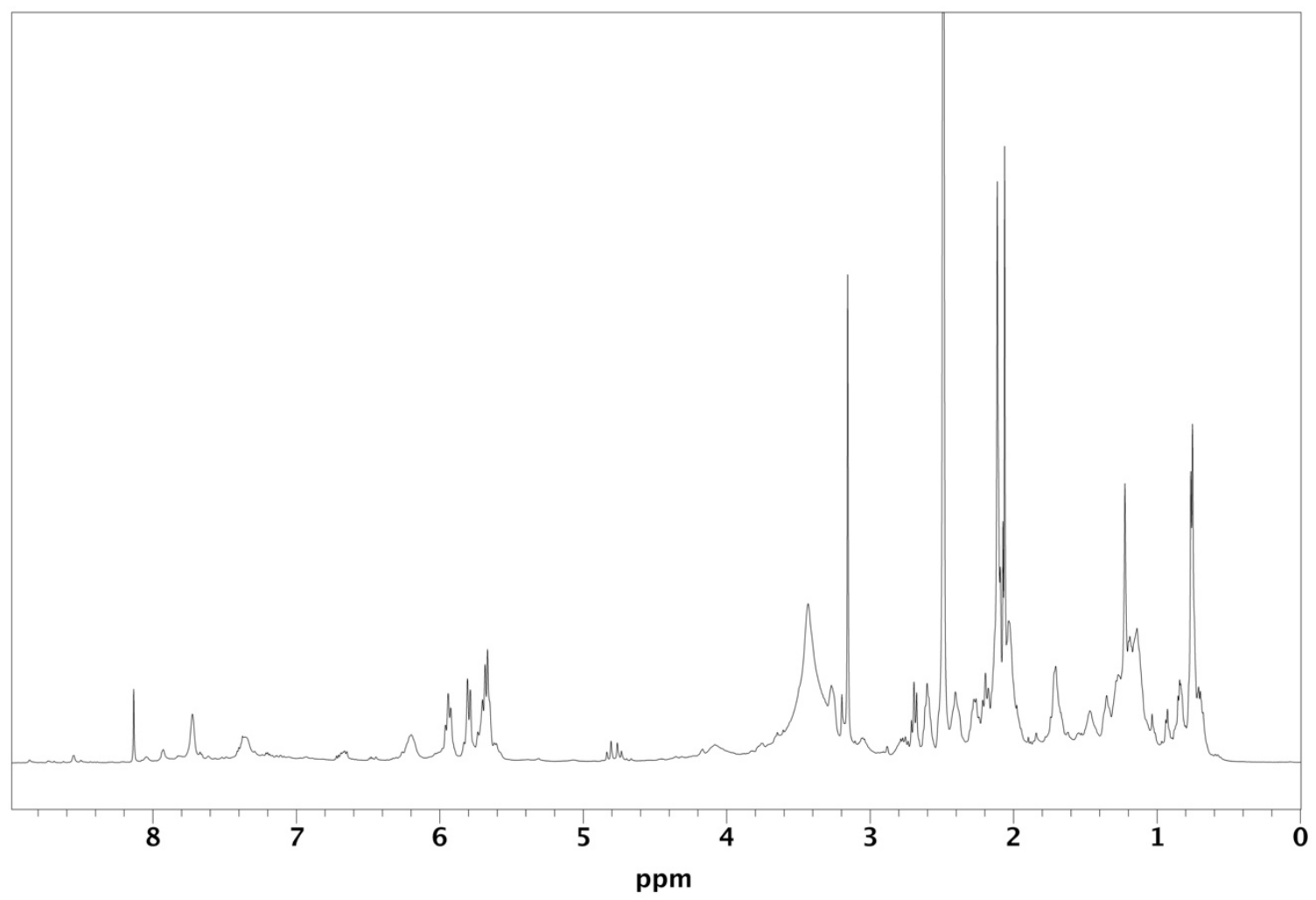

Figure S25. The ${ }^{1} \mathrm{H}-\mathrm{NMR}(\mathrm{DMSO}-d 6,600 \mathrm{MHz}$ ) spectrum of purified clifednamide A.

A.

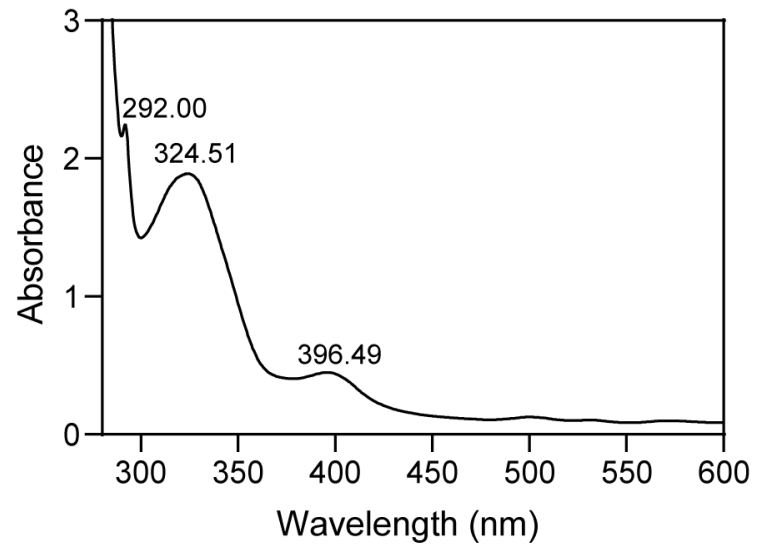

B.

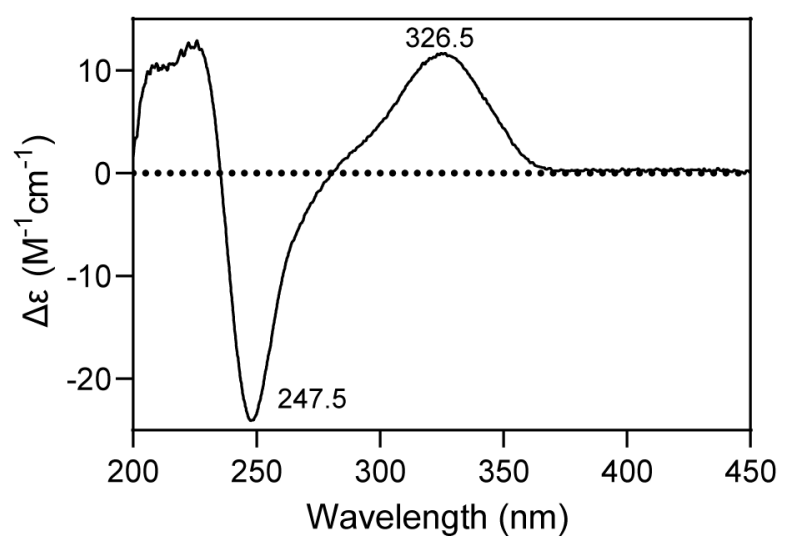

Figure S26. The UV-vis (panel A) and ECD (panel B) spectra of clifednamide C. 


\section{Acknowledgments}

The authors would like to thank Drs. Jeff Kao and Manmilan Singh (WUSTL Chemistry) for help with the acquisition of 2D NMR data. The authors thank Spencer Stumpf and Keshav K. Nepal (WUSTL Biology) for assistance with strain growth and acquisition of LC-MS/MS data. The authors also thank Dr. Deanna Mendez for the assistance with the CD spectroscopy experiments. High-resolution mass spectrometry experiments in this study made use of the NIH/NIGMS Biomedical Mass Spectrometry Resource at Washington University in St. Louis, MO, which is supported in part by the National Institute of General Medical Science within the National Institutes of Health through 5P41GM103422. Funding for this work was provided in part by the Sloan Foundation, Research Corporation for Science Advancement, and the Camille and Henry Dreyfus Foundation through Sloan Fellowship, Cottrell Scholar Award, and Camille Dreyfus Teacher Scholar Award to T. A. W. This work was also partially supported by the National Science Foundation under NSF-CAREER awards 1846005 to J. A. V. B. and 1654611 to T. A. W. 


\section{References}

(1) Qi, Y.; Ding, E.; Blodgett, J. A. V. Native and Engineered Clifednamide Biosynthesis in Multiple Streptomyces Spp. ACS Synth. Biol. 2018, 7 (2), 357-362.

https://doi.org/10.1021/acssynbio.7b00349.

(2) Jiao, Y. J.; Liu, Y.; Wang, H. X.; Zhu, D. Y.; Shen, Y. M.; Li, Y. Y. Expression of the Clifednamide Biosynthetic Pathway in Streptomyces Generates 27,28-Seco-Derivatives. $J$. Nat. Prod. 2020, 83 (9), 2803-2808. https://doi.org/10.1021/acs.jnatprod.0c00900.

(3) Greunke, C.; Antosch, J.; Gulder, T. A. M. Promiscuous Hydroxylases for the Functionalization of Polycyclic Tetramate Macrolactams-Conversion of Ikarugamycin to Butremycin. Chem. Commun. 2015, 51 (25), 5334-5336. https://doi.org/10.1039/c5cc00843c.

(4) Mak, P. J.; Denisov, I. G. Spectroscopic Studies of the Cytochrome P450 Reaction Mechanisms. Biochimica et Biophysica Acta - Proteins and Proteomics. Elsevier B.V. January 1, 2018, pp 178-204. https://doi.org/10.1016/j.bbapap.2017.06.021.

(5) Larkin, M. A.; Blackshields, G.; Brown, N. P.; Chenna, R.; Mcgettigan, P. A.; McWilliam, H.; Valentin, F.; Wallace, I. M.; Wilm, A.; Lopez, R.; et al. Clustal W and Clustal X Version 2.0. Bioinformatics 2007, 23 (21), 2947-2948. https://doi.org/10.1093/bioinformatics/btm404.

(6) Brown, N. P.; Leroy, C.; Sander, C. MView: A Web-Compatible Database Search or Multiple Alignment Viewer. Bioinformatics 1998, 14 (4), 380-381.

https://doi.org/10.1093/bioinformatics/14.4.380. 